BNL- 48907

UC-414

AGS/AD/93-5

INFORMAL

\title{
SPECTRUM ANALYSIS IN BEAM DIAGNOSTICS
}

\author{
S.Y. Zhang and W.T. Weng
}

April 23, 1993

\section{DISCLAIMER}

This report was prepared as an account of work sponsored by an agency of the United States Government. Neither the United States Government nor any agency thereof, nor any of their employees, makes any warranty, express or implied, or assumes any legal liability or responsibility for the accuracy, completeness, or usefulness of any information, apparatus, product, or process disclosed, or represents that its use would not infringe privately owned rights. Reference herein to any specific commercial product, process, or service by trade name, trademark, manufacturer, or otherwise does not necessarily constitute or imply its endorsement. recommendation, or favoring by the United States Government or any agency thereof. The views and opinions of authors expressed herein do not necessarily state or reflect those of the United States Government or any agency thereof.

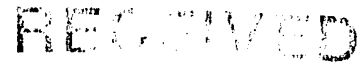

MAY 201903

$08 \% 1$

\section{ALTERNATING GRADIENT SYNCHROTRON DEPARTMENT}

\author{
BROOKHAVEN NATIONAL LABORATORY \\ ASSOCIATED UNIVERSITIES, INC. \\ UPTON, LONG ISLAND, NEW YORK
}

\section{UNDER CONTRACT NO. DE-AC02-76CHON016 WITH THE UNITED STATES DEPARTMENT OF ENERGY}




\title{
INSERT DISCLAIMER
}

\author{
SHEET HERE
}


Accelerator Division

Alternating Gradient Synchrotron Department

BROOKHAVEN NATIONAL LABORATORY

Upton, New York 11973

Accelerator Division

Technical Note

AGS/AD/Tech. Note No. 372

\section{SPECTRUM ANALYSIS IN BEAM DIAGNOSTICS}

S.Y. Zhang and W.T. Weng

April 7, 1993

ABSTRACT

In this article, we discuss fundamentals of the spectrum analysis in beam diagnostics, where several important particle motions in a circular accelerator are considered. The properties of the Fourier transform are presented. Then the coasting and the bunched beam motion in both longitudinal and transverse are studied. The discussions are separated for the signal particle, multiple particle, and the Schottky noise cases. To demonstrate the interesting properties of the beam motion spectrum, time domain functions are generated, and then the associated spectra are calculated and plotted. In order to show the whole picture in a single plot, some data have been scaled, therefore they may not be realistic in an accelerator. 


\section{Introduction}

In this article, we present fundamentals of the spectrum analysis in the beam diagnostics, which is a very useful method in the analysis of the beam dynamics. Interesting discussions can be found in $[1,2,4,6]$.

We consider circular particle accelerators. The beam can be unbunched or bunched, the motion of the beam is in both the longitudinal and the transverse. The motions of the particles in the beam can be coherent or incoherent, and these particles can be in various distributions. Moreover, due to the machine imperfections, the space charge, and the beam-beam effects, the various motions are correlated in one way or another.

We first discuss properties of the Fourier transform, the Fourier transform for periodic functions, and the amplitude and energy spectra. The results are used to study the spectrum for most important cases in beam diagnostics. The single particle case will be studied first, which includes the longitudinal and transverse motions, and each aspect is separated for coasting and bunched beams, respectively. Then, we consider the multiple particles, which can be distributed in different ways, but the particles are assumed to move together. Finally, under the assumption that the particles move independently, the Schottky noise is studied.

Each spectrum analysis in the article represents some typical case. To demonstrate the relation between the time domain function and the frequency spectrum, time domain functions are generated for each case, and the corresponding frequency spectra are generated by discrete Fourier transform (DFT). To present a clear view of the spectrum, the data in some examples have to be compromised, therefore the spectrum shown in this article may not be realistic, which inplies that the ratio between different frequency components, the bandwidth of the frequency bands, and the frequency span for different motions may be scaled to show the whole picture in a single plot for some cases. 


\section{Preliminary}

\section{2-1. Fourier Transform}

The Fourier transform of a time domain function $f(t)$ is

$$
F(\omega)=\int_{-\infty}^{\infty} f(t) e^{-j \omega t} d t
$$

where $F(\omega)$ is the frequency spectrum. In turn, the time domain function $f(t)$ can be represented by the frequency spectrum $F(\omega)$ as

$$
f(t)=\frac{1}{2 \pi} \int_{-\infty}^{\infty} F(\omega) e^{j \omega t} d \omega
$$

In the follows, we present two useful properties of the Fourier transform in the spectrum analysis of beam diagnostics.

The first property is the amplitude modulation of $f(t)$. The following Fourier pair

$$
f(t) e^{j \omega_{0} t} \rightarrow F\left(\omega-\omega_{0}\right)
$$

can be easily proved by using (2-1). For a real amplitude modulation of $f(t)$ by $\cos \omega_{0} t$, we have the follows,

$$
f(t) \cos \omega_{0} t \rightarrow \frac{1}{2}\left[F\left(\omega-\omega_{0}\right)+F\left(\omega+\omega_{0}\right)\right]
$$

The second property is the time modulation of $f(t)$ by $\tau_{1}$, which is shown as,

$$
f\left(t+\tau_{1}\right) \rightarrow e^{j \omega \tau_{1}} F(\omega)
$$

If the modulation function is,

$$
\tau_{1}=\tau \sin \phi
$$

then the right side of (2-5) can be calculated by using [3],

$$
e^{j \omega \tau \sin \phi}=\sum_{k=-\infty}^{\infty} J_{k}(\omega \tau) e^{j k \phi}
$$

where $k$ is an integer, and $J_{k}$ is the Bessel function of order $k$, which are shown in Fig.1. 


\section{2-2. Fourier Transform of Periodic Functions}

The periodic function with period $T$,

$$
f(t)=f(t-T)
$$

can be represented by the harmonics at $n \omega_{0}, \omega_{0}=2 \pi / T$, as,

$$
f(t)=\sum_{n=-\infty}^{\infty} a_{n} e^{j n \omega_{0} t}
$$

where

$$
a_{n}=\frac{1}{T} \int_{-T / 2}^{T / 2} f(t) e^{-j n \omega_{0} t} d t
$$

Equation $(2-10)$ can be proved by using the orthogonality of the function $e^{j \omega t}$, which is

$$
\frac{1}{T} \int_{-T / 2}^{T / 2} e^{j(n-m) \omega_{0} t} d t= \begin{cases}0, & n \neq m \\ 1, & n=m\end{cases}
$$

where $n$ and $m$ are integers. By multiplying both sides of (2-9) by $e^{-j m \omega_{0} t}$, and averaging over one period of $T,(2-10)$ can be proved using (2-11).

Consider a periodic function

$$
f(t)=\sum_{n=-\infty}^{\infty} \delta(t-n T)
$$

where $\delta$ represents a delta function. Let $f(t)$ be represented by $(2-9)$, then from $(2-10)$ we have

$$
a_{n}=\frac{1}{T} \int_{-T / 2}^{T / 2} \delta(t) e^{-j n \omega_{0} t} d t=\frac{1}{T}=\frac{\omega_{0}}{2 \pi}
$$

which shows that $f(t)$ in (2-12) can be written as,

$$
f(t)=\frac{\omega_{0}}{2 \pi} \sum_{n=-\infty}^{\infty} e^{j n \omega_{0} t}
$$

Combining (2-12) and (2-14), we get an important equation, 


$$
\sum_{n=-\infty}^{\infty} \delta(t-n T)=\frac{\omega_{0}}{2 \pi} \sum_{n=-\infty}^{\infty} e^{j n \omega_{0} t}
$$

Furthermore, if we take the Fourier transform for both sides of (2-15), we have the following equations,

$$
F(\omega)=\int_{-\infty}^{\infty} \sum_{n=-\infty}^{\infty} \delta(t-n T) e^{-j \omega t} d t=\sum_{n=-\infty}^{\infty} e^{-j \omega n T}=\sum_{n=-\infty}^{\infty} e^{-j 2 \pi n \omega / \omega_{0}}
$$

and

$$
F(\omega)=\frac{\omega_{0}}{2 \pi} \int_{-\infty}^{\infty} \sum_{n=-\infty}^{\infty} e^{j n \omega_{0} t} e^{-j \omega t} d t=\omega_{0} \sum_{n=-\infty}^{\infty} \delta\left(\omega-n \omega_{0}\right)
$$

In the last step of (2-17), we have used another identity of the orthogonality of the function $e^{-j\left(\omega-n \omega_{0}\right) t}$, i.e., $[5]$,

$$
F(1)=\int_{-\infty}^{\infty} e^{-j \omega t} d t=2 \pi \delta(\omega)
$$

From (2-16) and (2-17), we get another important equation,

$$
\sum_{n=-\infty}^{\infty} e^{-j 2 \pi n \omega / \omega_{0}}=\omega_{0} \sum_{n=-\infty}^{\infty} \delta\left(\omega-n \omega_{0}\right)
$$

In general, for a periodic function

$$
f_{1}(t)=f_{1}(t-T)
$$

if

$$
f(t)=\sum_{n=-\infty}^{\infty} f_{1}(t-n T)
$$

then we have

$$
\begin{gathered}
F(\omega)=\int_{-\infty}^{\infty} \sum_{n=-\infty}^{\infty} f_{1}(t-n T) e^{-j \omega t} d t=\sum_{n=-\infty}^{\infty} \int_{-\infty}^{\infty} f_{1}(t-n T) e^{-j \omega(t-n T)} e^{-j \omega n T} d(t-n T) \\
=\sum_{n=-\infty}^{\infty} F_{1}(\omega) e^{-j \omega n T}=\omega_{0} F_{1}(\omega) \sum_{n=-\infty}^{\infty} \delta\left(\omega-n \omega_{0}\right)
\end{gathered}
$$

where $F_{1}(\omega)$ is the Fourier transform of $f_{1}(t)$, and in the last step we have used (2-19). 
The equation (2-22) shows that the overall frequency spectrum of a periodic function $f(t)$ is simply a sampling of the spectrum of the signal $f_{1}(t)$. The envelope of $F(\omega)$ is determined by $F_{1}(\omega)$, while the sampling rate is determined by $T$.

\section{2-3. Amplitude and Energy Spectra}

The Parseval's theorem [5] shows that the energy of $f(t)$ can be determined from the corresponding frequency spectrum $F(\omega)$,

$$
\int_{-\infty}^{\infty} f^{2}(t) d t=\frac{1}{2 \pi} \int_{-\infty}^{\infty}|F(\omega)|^{2} d \omega
$$

where $|F(\omega)|$ is called the amplitude spectrum, and $|F(\omega)|^{2}$ is called the energy spectrum.

In the beam signal measurement, the power of the signal is often of interest. The power of the time function $f(t)$ can be described by the energy spectrum as follows,

$$
P=\lim _{r \rightarrow \infty} \frac{1}{2 \tau} \int_{-\tau}^{\tau} f^{2}(t) d t=\lim _{r \rightarrow \infty} \frac{1}{4 \pi \tau} \int_{-\infty}^{\infty}|F(\omega)|^{2} d \omega
$$

It is often of interest to know the power $P_{\alpha}$ contained in a frequency baud, with a center frequency $\omega_{\alpha}$ and a frequency $\operatorname{span} \Delta \omega$,

$$
\omega_{\alpha}-\Delta \omega / 2 \leq \omega<\omega_{\alpha}+\Delta \omega / 2
$$

This power can be shown as,

$$
P_{\alpha}=\lim _{r \rightarrow \infty} \frac{1}{2 \tau} \int_{-r}^{r} f_{\alpha}^{2}(t) d t=\lim _{r \rightarrow \infty} \frac{1}{2 \pi r} \int_{\omega_{a}-\Delta \omega / 2}^{\omega_{a}+\Delta \omega / 2}|F(\omega)|^{2} d \omega
$$

where $f_{a}(t)$ is the associated time domain function. Considering that $|F(\omega)|^{2}$ is the same for positive and negative frequencies, the factor $1 /(4 \pi \tau)$ in $(2-24)$ becomes $1 /(2 \pi \tau)$ in (2-26). Letting $\Delta \omega \rightarrow 0,(2-26)$ shows that the power of the time domain function $f_{\alpha}(t)$ is proportional to the energy spectrum $|F(\omega)|^{2}$. The process of the particle motion in an accelerator can be assumed to be stationary, i.e., the process is not time dependent. 
Therefore, an amplitude or energy spectrum can provide useful information for the beam diagnostics.

In this article, the spectrum analysis of the beam diagnostics is shown by either the amplitude spectrum or the energy spectrum, depending on the requirement of the plot. In general the amplitude spectrum is used, however, if the ratio of the useful signal and the noises of the amplitude spectrum is small, the energy spectrum will be used.

\section{Single Particle}

\section{3-1. Longitudinal}

Let the revolution period of the particle in a circular accelerator be $T$, then the revolution frequency is

$$
\omega_{0}=\frac{2 \pi}{T}
$$

The single unit charge particle signal is,

$$
f(t)=\sum_{n=-\infty}^{\infty} \delta(t-n T)
$$

In Fig.2a we show three signals with the same amplitude and different period, i.e., $T$ equals $0.3125 \mu s$ for $r_{1}, 0.625 \mu s$ for $r_{2}$, and $1.25 \mu s$ for $r_{3}$.

Using (2-15) and (2-17), the frequency spectrum of $f(t)$ is found,

$$
F(\omega)=\omega_{0} \sum_{n=-\infty}^{\infty} \delta\left(\omega-n \omega_{0}\right)
$$

which shows that the frequency spectrum of $f(t)$ is also a train of delta functions, the distance between pulses is $\omega_{0}$, and the amplitude of the spectrum is determined by a factor of $\omega_{0}$. For the time domain signals shown in Fig.2a, the frequency spectra $F(\omega)$ are shown in Fig.2b. Note that $\omega_{b}$ of the spectra $R_{1}, R_{2}$, and $R_{3}$ are $3.2 \mathrm{MHz}, 1.6 \mathrm{MHz}$, and $0.8 \mathrm{MHz}$, respectively. The amplitudes of the spectra also vary accordingly. 
One may find the revolution frequency $\omega_{0}$, or the revolution period $T$, from the frequency measurement of the beam signal.

\section{3-2. Transverse}

The single particle transverse signal can be modeled as an amplitude modulation on the signal of (3-1) as,

$$
f(t)=\cos \nu_{1} \omega_{0} t \sum_{n=-\infty}^{\infty} \delta(t-n T)
$$

where $\nu_{1}$ is the non-in teger part of the betatron tune. In Fig.3a, the signals $r_{1}, r_{2}$ and $r_{3}$, which are modulated with $\nu_{1}$ equal $0,0.125$ and 0.25 , respectively, are shown.

Using (2-4) and (3-3), the frequency spectrum is readily shown as,

$$
\begin{gathered}
F(\omega)=\frac{1}{2}\left[F\left(\omega-\nu_{1} \omega_{0}\right)+F\left(\omega+\nu_{1} \omega_{0}\right)\right] \\
=\frac{\omega_{0}}{2}\left[\sum_{n=-\infty}^{\infty} \delta\left(\omega-n \omega_{0}-\nu_{1} \omega_{0}\right)+\sum_{n=-\infty}^{\infty} \delta\left(\omega-n \omega_{0}+\nu_{1} \omega_{0}\right)\right]
\end{gathered}
$$

For each delta pulse originally located at $\omega=n \omega_{0}$, the frequency spectrum becomes two pulses located at $n \omega_{0}-\nu_{1} \omega_{0}$ and $n \omega_{0}+\nu_{1} \omega_{0}$, and the amplitudes are reduced to half. The distances of the betatron lines from $n \omega_{0}$, by $\pm \nu_{1} \omega_{0}$, are not dependent on $n$. The amplitude spectra for the time signals shown in Fig.3a are shown in Fig.3b, where the frequency is indicated by the harmonics of the signals.

The information of the betatron tune is contained in the betatron lines.

In general, a closed orbit error or a deviation from the nominal energy can introduce a DC modulation for $f(t)$, and the modulation signal in (3-4), $\cos \nu_{1} \omega_{0} t$, is replaced by $a+\cos \nu_{1} \omega_{0} t$, where a represents the combined DC modulation. The time domain signals are shown in Fig.3c, where $r_{2}$ and $r_{3}$ are modulated by the same DC signal $a$, but different frequencies as that in Fig.3a. In Fig.3d, the amplitude spectra are shown. Note that the betatron lines are not changed, and the pulses at $n \omega_{0}$ provides information on 
the DC modulation $a$.

\section{3-3. Bunched, Longitudinal}

By 'bunched' we imply that the RF system is on, and therefore there will exist synchrotron oscillations. This is modeled as a time modulation of $f(t)$ by,

$$
\tau_{1}=\tau \sin \omega_{S} t
$$

where $\omega_{S}$ is the synchrotron frequency. The time signal therefore can be written as,

$$
f(t)=\sum_{n=-\infty}^{\infty} \delta\left(t-n T+\tau \sin \omega_{S} n T\right)
$$

One signal without modulation and two signals modulated by the same frequency, but different amplitudes, are shown in Fig.4a, where the amplitude of the modulation signal of $r_{3}$ is twice of the one for $r_{2}$.

Using (2-16), the spectrum is found,

$$
F(\omega)=\sum_{n=-\infty}^{\infty} e^{-j \omega\left(n T-\tau \sin \omega_{S} n T\right)}=\sum_{n=-\infty}^{\infty} e^{j \omega \tau \sin 2 \pi n \omega_{\mathcal{S}} / \omega_{0}} e^{-j \omega 2 \pi n / \omega_{0}}
$$

Using (2-7), the equation (3-8) is written as,

$$
\begin{aligned}
F(\omega) & =\sum_{k=-\infty}^{\infty} J_{k}(\omega \tau) \sum_{n=-\infty}^{\infty} e^{j k 2 \pi n \omega_{S} / \omega_{0}} e^{-j \omega 2 \pi n / \omega_{0}} \\
& =\omega_{0} \sum_{k=-\infty}^{\infty} J_{k}(\omega \tau) \sum_{n=-\infty}^{\infty} \delta\left(\omega-n \omega_{0}-k \omega_{S}\right)
\end{aligned}
$$

where in the last step we have used (2-19).

The energy spectra of the signals shown in Fig.4a are shown in Fig.4b. We note that in the energy spectra, instead of the original single pulse at $n \omega_{0}$, there is a synchrotron satellite, each synchrotron line is distanced from the adjacent one by the synchrotron frequency $\omega_{S}$. The 0th order synchrotron lines locate exactly at the harmonics of $n \omega_{0}$ 's, their amplitude are determined by $J_{0}$. The $k$ th order syachrotron lines are distanced from $n \omega_{0}$ by $\pm k \omega_{s}$, and their amplitude are determined by $s_{k}$. When $\omega r$ is sub- 
stantiaily smaller than $k$, the $k$ th sidebands will have small amplitude, which can be observed in Fig.4b.

From the measurement, we may directly find the synchrotron frequency. By matching the Bessel function curves, one may also find the synchrotron oscillation amplitude $\tau$.

\section{3-4. Bunched, Transverse}

In the bunched beam transverse signal, the amplitude modulation is combined by a time modulation. The signal is,

$$
f(t)=\cos \nu_{1} \omega_{0} t \sum_{n=-\infty}^{\infty} \delta\left(t-n T+\tau \sin \omega_{S} n T\right)
$$

One original signal $r_{1}$ and two signals modulated with same $\omega_{S}$, but different $\nu_{1}$ and $\tau$. are shown in Fig.5a, where $\nu_{1}$ and $\tau$ of $r_{3}$ are twice of that of $r_{2}$. By using (3-5) and (39), the frequency spectrum can be written as,

$$
\begin{aligned}
F(\omega) & =\frac{\omega_{0}}{2}\left[\sum_{k=-\infty}^{\infty} J_{k}\left(\omega \tau-\nu_{1} \omega_{0} \tau\right) \sum_{n=-\infty}^{\infty} \delta\left(\omega-n \omega_{0}-k \omega_{S}-\nu_{1} \omega_{0}\right)\right. \\
+ & \left.\sum_{k=-\infty}^{\infty} J_{k}\left(\omega \tau+\nu_{1} \omega_{0} \tau\right) \sum_{n=-\infty}^{\infty} \delta\left(\omega-n \omega_{0}-k \omega_{S}+\nu_{1} \omega_{0}\right)\right]
\end{aligned}
$$

The energy spectra of the signals shown in Fig.5a are shown in Fig.5b. We observe that the distance of the two betatron lines at each harmonic of $R_{3}$ is twice of that of $R_{2}$.

For each betatron line, there is a synchrotron satellite. The amplitude of the synchrotron satellites are determined by $\omega_{0} / 2$ and $J_{k}\left(\omega \tau \pm \nu_{1} \omega_{0} \tau\right)$.

After the synchrotron lines are identified from the betatron lines, one may find the synchrotron and betatron frequencies.

\section{Multiple Particles}

The beam with short bunches may be considered as the single particle. In general, the particle distribution has to be considered if the bunches are not very short. There are 
many possible particle distributions, such as Gaussian, Lorentzian, parabolic, etc. In this section, we take the Gaussian distribution as example, for others, similar means can be applied.

Consider the Gaussian distribution in time domain,

$$
f_{1}(t)=e^{-\sigma^{2} t^{2}}
$$

which is shown in Fig.6a, where the unity amplitude and the standard deviation of $1 /\left(2^{1 / 2} \sigma\right)$ are also shown. Note that the full bunch length is 4 times of the standard deviation, i.e., $\tau_{L}=2 \times 2^{1 / 2} / \sigma$.

The frequency spectrum is 3$]$,

$$
F_{1}(\omega)=\frac{\pi^{1 / 2}}{\sigma} e^{-\omega^{2} / 4 \sigma^{2}}
$$

which is shown in Fig. $6 \mathrm{~b}$, also shown are the amplitude $\pi^{1 / 2} / \sigma$, and the effective bandwidth $2^{1 / 2} \sigma$.

\section{4-1. Longitudinal}

We consider the periodic function with the period $T$ and eash pulse of the function with the Gaussian distribution $f_{2}(t)$,

$$
f(t)=\sum_{n=-\infty}^{\infty} f_{1}(t-n T)=\sum_{n=-\infty}^{\infty} e^{-\sigma^{2}(t-n T)^{2}}
$$

Two such functions with different period are shown in Fig.7a.

Using equations (2-22) and (4-2), the frequency spectrum is readily written as,

$$
F(\omega)=\omega_{0} \sum_{n=-\infty}^{\infty} F_{1}(\omega) \delta\left(\omega-n \omega_{0}\right)=\frac{\pi^{1 / 2} \omega_{0}}{\sigma} \sum_{n=-\infty}^{\infty} e^{-\omega^{2} / 4 \sigma^{2}} \delta\left(\omega-n \omega_{0}\right)
$$

The amplitude spectra for the functions shown in Fig.7a are shown in Fig.7b. The the spectrum has a same envelope as the spectrum of $F_{1}(\omega)$ of $f_{1}(t)$, but it is sampled with the frequency period of $\omega_{0}=2 \pi / T$. Also the amplitude is also changed by a factor of $\omega_{0}$. 
In addition to the information of $\omega_{0}$, from the shape of the spectrum envelope, one may find the particle distribution, and from the bandwidth of the spectrum envelope, one may find the bunch length.

\section{4-2. Transverse}

In this case the time function is,

$$
f(t)=\cos \nu_{1} \omega_{0} t \sum_{n=-\infty}^{\infty} e^{-\sigma^{2}(t-n T)^{2}}
$$

and using (3-5) and (4-4) the spectrum is found,

$$
\begin{aligned}
F(\omega) & =\frac{\pi^{1 / 2} \omega_{0}}{2 \sigma}\left[\sum_{n=-\infty}^{\infty} e^{-\left(\omega-\nu_{1} \omega_{0}\right)^{2} / 4 \sigma^{2}} \delta\left(\omega-n \omega_{0}-\nu_{1} \cdot \omega_{0}\right)\right. \\
& \left.+\sum_{n=-\infty}^{\infty} e^{-\left(\omega+\nu_{1} \omega_{0}\right)^{2} / 4 \sigma^{2}} \delta\left(\omega-n \omega_{0}+\nu_{1} \omega_{0}\right)\right]
\end{aligned}
$$

The amplitude spectra of the two modulated time domain signals on $r_{1}$ and $r_{2}$ in Fig.7a. by the same betatron frequency, are shown in Fig.8. Note that the shape of the overall spectrum is still the Gaussian, the amplitude of the two betatron line for each harmonic are modulated by $e^{-\left(\omega-\nu_{1} \omega_{0}\right)^{2} / 4 \sigma^{2}}$ and $e^{-\left(\omega+\nu_{1} \omega_{0}\right)^{2} / 4 \sigma^{2}}$, respectively. Therefore, they only differ by a frequency shift.

The information contained are the betatron frequency, the time domain distribution, and the bunch length.

\section{4-3. Bunched, Longitudinal}

The time domain function is,

$$
f(t)=\sum_{n=-\infty}^{\infty} e^{-\sigma^{2}\left(t-n T+\tau \sin \omega_{S} n T\right)^{2}}
$$

and using (3-9) and (4-4) the spectrum is found,

$$
F(\omega)=\frac{\pi^{1 / 2} \omega_{0}}{\sigma} \sum_{k=-\infty}^{\infty} J_{k}(\omega \tau) e^{-\omega^{2} / 4 \sigma^{2}} \sum_{n=-\infty}^{\infty} \delta\left(\omega-n \omega_{0}-k \omega_{S}\right)
$$


The amplitude spectra of the modulated time domain signals on $r_{1}$ and $r_{2}$ in Fig.7a, by the same synchrotron frequency $\omega_{S}$ and amplitude $\tau$, are shown in Fig.9. The amplitude of the synchrotron lines are determined by both the Bessel function $J_{k}(\omega \tau)$ and the Gaussian envelope $e^{-\omega^{2} / 4 \sigma^{2}}$. Therefore, for the pulses located at the harmonics, the amplitude is determined by $J_{0}(\omega \tau)$ and $e^{-\omega^{2} / 4 \sigma^{2}}$, which can be observed in Fig.9. For the amplitudes of the first and second synchrotron lines, the influence of $J_{1}(\omega \tau)$ and $J_{2}(\omega \tau)$ can also be observed.

\section{4-4. Bunched, Transverse}

In this case the time domain function is,

$$
f(t)=\cos \nu_{1} \omega_{0} t \sum_{n=-\infty}^{\infty} e^{-\sigma^{2}\left(t-n, T+r \sin \omega_{S} n T\right)^{2}}
$$

and using (2-4) and (4-8) the spectrum is found,

$$
\begin{aligned}
F(\omega)= & \frac{\pi^{1 / 2} \omega_{0}}{2 \sigma}\left[\sum_{k=-\infty}^{\infty} J_{k}\left(\omega \tau-\nu_{1} \omega_{0} \tau\right) e^{-\left(\omega-\nu_{1} \omega_{0}\right)^{2} / 4 \sigma^{2}} \sum_{n=-\infty}^{\infty} \delta\left(\omega-n \omega_{0}-k \omega_{S}-\nu_{1} \omega_{0}\right)\right. \\
& \left.+\sum_{k=-\infty}^{\infty} J_{k}\left(\omega \tau+\nu_{1} \omega_{0} \tau\right) e^{-\left(\omega+\nu_{1} \omega_{0}\right)^{2} / 4 \sigma^{2}} \sum_{n=-\infty}^{\infty} \delta\left(\omega-n \omega_{0}-k \omega_{S}+\nu_{1} \omega_{0}\right)\right]
\end{aligned}
$$

The amplitude spectra of the modulated signals on $r_{1}$ and $r_{2}$ in Fig.7a, by the same betatron and the same synchrotron frequencies, are shown in Fig.10.

\section{Schottky Noise}

In the last section, we have assumed that in a bunch all particles move altogether, i.e., there are no spread of momentum, and no spread of betatron and synchrotron frequencies. In reality, more or less the particles are moving independently. The situation can be represented as the Schottky noise [1]. 


\section{5-1. Longitudinal}

We assume that the particles move in slightly different revolution times $T_{i}$. Let there be $N$ particles. The time domain signal is,

$$
f(t)=\sum_{i=1}^{N} \sum_{n=-\infty}^{\infty} \delta\left(t-n T_{i}\right)
$$

From (3-3) the frequency spectrum is simply,

$$
F(\omega)=\sum_{i=1}^{N} \omega_{i} \sum_{n=-\infty}^{\infty} \delta\left(\omega-n \omega_{i}\right)
$$

Where $\omega_{i}=2 \pi / T_{i}$, and we assume that the distribution of the deviation of $T_{i}$ from $T$, therefore the deviation of $\omega_{i}$ from $\omega_{0}$, are random and small.

To find the bandwidth of the frequency band at the $n$th harmonic, we let

$$
\Delta \omega / 2=\max \left\{\left|\omega_{i}-\omega_{0}\right|\right\}
$$

We also define a $\Lambda$ function of the variable of the delta function in (5-2) as,

$$
\Lambda\left(\omega-n \omega_{i}\right)=\omega-n\left(\omega_{0}+\Delta \omega / 2\right)=\omega-n \omega_{0}-n \Delta \omega / 2
$$

which indicates that the amplitude spectrum of the $n$th harmonic is centered at $n \omega_{0}$, with the half bandwidth $n \Delta \omega / 2$, and therefore the bandwidth $n \Delta \omega$. It is clear that the bandwidth of the power spectrum will be larger as $n$ becomes larger.

In the next, we discuss the energy contained in each harmonic band. Here the relative phase between the particles must be considered. We assume that the particles are randomly distributed in azimuth, therefore the equation (5-1) becomes,

$$
f(t)=\sum_{i=1 n}^{N} \sum_{=-\infty}^{\infty} \delta\left(\left(t+t_{i}\right)-n T_{i}\right)
$$

where $t_{i}$ represents the time, and therefore the azimuth, distribution of the $i$ th particle. Using (2-5) the frequency spectrum becomes,

$$
F(\omega)=\sum_{i=1}^{N} \omega_{i} e^{j \omega t_{i}} \sum_{n=-\infty}^{\infty} \delta\left(\omega-n \omega_{i}\right)
$$


Consider only the $n$th harmonic. Taking the average of the energy spectrum over the particles, we have,

$$
\begin{aligned}
& \left.<|F(\omega)|^{2}\right\rangle_{n}=\left\langle F(\omega) F^{*}(\omega)\right\rangle_{n}=\left\langle\sum_{i=1}^{N} \sum_{p=1}^{N} \omega_{i} \omega_{p} \delta\left(\omega-n \omega_{i}\right) \delta\left(\omega-n \omega_{p}\right) e^{j \omega t_{i}} e^{-j \omega t_{p}}\right\rangle \\
& =\left\langle\sum_{i=1}^{N} \sum_{p=1}^{N} \omega_{i} \omega_{p} \delta\left(\omega-n \omega_{i}\right) \delta\left(\omega-n \omega_{p}\right) e^{j \omega\left(t_{i}-t_{p}\right)}\right\rangle=\left\langle\sum_{i=1}^{N} \omega_{i}^{2} \delta\left(\omega-n \omega_{i}\right)\right\rangle \approx \omega_{0}^{2} N \quad(5-7)
\end{aligned}
$$

where the symbol \langle\rangle$_{n}$ denotes the averaging over the $n$th harmonic, and $F^{*}(\omega)$ is the conjugate of $F(\omega)$. In the fourth step of (5-7), we have used the fact that for many randomly distributed particles in azimuth, the averaged phase factor is zero unless $t_{i}=t_{p}$, which eliminates one summation. In this equation, we also assumed temporarily that $\omega_{i}=\omega_{p}$.

This result can be easily extended to the case where $\omega_{i}=\omega_{p}$ is not required, i.e., there exists a frequency band instead of a pulse at the $n$th harmonic. The particles can be separated into, say, $M$ groups. In the $m$ th group, there are $N_{m}$ particles, whose revo lution frequency is the same. The equation (5-7) can be used to evaluate the energy of the particles in each group, which equals $\omega_{0}^{2} N_{m}$ for $m$ th group. Since the frequencies between the groups are not overlapped, the total energy in the band is simply,

$$
\left.<|F(\omega)|^{2}\right\rangle_{n}=\omega_{0}^{2} \sum_{m=1}^{M} N_{m}=\omega_{0}^{2} N
$$

The equations (5-7) and (5-8) show that if the phases of the particles are randomly distributed, the energy contained in a narrow band is proportional to the total number of the particles $N$, because of the phase factor cancellation. Also the energy contained in each band is independent of $n$.

In the beam spectrum observation, both positive and negative frequencies contribute. Therefore, if the real measurement is considered a factor of 2 should be multiplied to the results shown in (5-7) and (5-8), 
In Fig.11, the energy spectrum of the longitudinal Schottky noise is shown. The amplitude spectrum could be used, however, since the ratio of the useful signal to the noises in the DFT is not large, the energy spectrum provides a clearer picture. Note that the energy contained in each harmonic band is approximately the same. Consider that the bandwidth of the bands is larger when $n$ becomes larger, the amplitude of the energy spectrum will be smaller as $n$ becomes larger. If $n$ is even larger, the energy spectrum will eventually overlap.

In general, the frequency spread is due to the particle momentum spread, therefore using the relation,

$$
\Delta \omega=-\omega_{0} \eta \frac{\Delta p}{p}
$$

where $\eta$ is the frequency slip factor, we can find the momentum spread $\Delta p / p$ from the frequency spread in the spectrum.

\section{5-2. Transverse}

Let $\nu_{0}$ and $\nu_{i}$ be the non-integer parts of the betatron tune corresponding to $\omega_{0}$ and $\omega_{i}$, respectively, and let $\nu$ be the betatron tune corresponding to $\omega_{0}$. The time domain signal is,

$$
f(t)=\sum_{i=1 n}^{N} \sum_{-\infty}^{\infty} \cos \left(2 \pi \nu_{i} t / T_{i}\right) \delta\left(t-n T_{i}\right)
$$

Using (3-5) and (5-2) the frequency spectrum is found to be,

$$
\begin{gathered}
F(\omega)=\sum_{i=1}^{N} \sum_{n=-\infty}^{\infty} \frac{\omega_{i}}{2}\left[\delta\left(\omega-n \omega_{i}-\nu_{i} \omega_{i}\right)+\delta\left(\omega-n \omega_{i}+\nu_{i} \omega_{i}\right)\right] \\
\approx \frac{\omega_{0}}{2} \sum_{i=1}^{N} \sum_{n=-\infty}^{\infty}\left[\delta\left(\omega-n \omega_{i}-\nu_{i} \omega_{i}\right)+\delta\left(\omega-n \omega_{i}+\nu_{i} \omega_{i}\right)\right]
\end{gathered}
$$

which shows that for the $n$th harmonic, the frequency band splits into two sidebands at $\left(n \pm \nu_{0}\right) \omega_{0}$ 
To find the bandwidth of each band, we need the follows. Let $\Delta \nu$ be the spread of betatron tune, which comes from the particle momentum spread. We have

$$
\frac{\Delta v}{\nu}=\xi \frac{\Delta p}{p}
$$

where $\xi$ is the machine chromaticity. Combined with (5-9), the betatron tune spread is related to the revolution frequency spread by,

$$
\Delta \nu=-\frac{\xi \nu}{\eta} \frac{\Delta \omega}{\omega_{0}}
$$

Using the definition of (5-3), we consider the half bandwidth of the first sideband in the $n$th harmonic, represented by the variable in the first delta function in (5-11),

$$
\begin{gathered}
\Lambda\left(\omega-n \omega_{i}-\nu_{i} \omega_{i}\right)=\omega-n\left(\omega_{0}+\Delta \omega / 2\right)-\left(\nu_{0}-\Delta \nu / 2\right)\left(\omega_{0}+\Delta \omega / 2\right) \\
\approx \omega-\left(n-\nu_{0}\right) \omega_{0}-\left(n \Delta \omega / 2-\omega_{0} \Delta \nu / 2+\nu_{0} \Delta \omega / 2\right)
\end{gathered}
$$

Note that in this equation, we have used the fact that the frequency spread of $\omega_{0}+\Delta \omega / 2$ is associated with the betatron tune spread of $\nu_{0}-\Delta y / 2$, which is shown in (5-13). Also using (5-13), the equation (5-14) becomes,

$$
\Lambda\left(\omega-n \omega_{i}-\nu_{i} \omega_{i}\right) \approx \omega-\left(n-\nu_{0}\right) \omega_{0}-\left(n+\frac{\xi \nu}{\eta}+\nu_{0}\right) \Delta \omega / 2
$$

For another sideband, we have,

$$
\Lambda\left(\omega-n \omega_{i}+\nu_{i} \omega_{i}\right) \approx \omega-\left(n+\nu_{0}\right) \omega_{0}-\left(n-\frac{\xi \nu}{\eta}-\nu_{0}\right) \Delta \omega / 2
$$

which shows that for the $n$th harmonic the two sidebands are approximately at $\left(n+\nu_{0}\right) \omega_{0}$, with the bandwidth $\left(n \pm \frac{\xi \nu}{\eta} \pm \nu_{0}\right) \Delta \omega$, respectively, i.e., the bandwidth of the two sidebands are different. If $n$ becomes larger, the bandwidth of both sidebands will be larger, but the difference between the two bandwidth stays the same, which is $2\left(\frac{\xi \nu}{\eta}+\nu_{0}\right) \Delta \omega$.

Using the same approach as that in (5-5), (5-6), (5-7), and (5-8), the energy contained in one of the sidebands, which is denoted by $n 1$, can be found as, 


$$
<|F(\omega)|^{2}>_{n 1} \approx\left(\frac{\omega_{0}}{2}\right)^{2} N
$$

which indicates that the energy contained in the two sidebands are the same, and also they are not dependent on $n$, which is shown in Fig.12.

One may find $\nu_{0}$ by allocating the sideband center. Then the frequency spread $\Delta \omega$ can be found by using (5-15). Using (5-13), the betatron tune spread $\Delta \nu$ can be found.

If the betatron tunes of the particles are with different amplitude $a_{i}$ and different initial phase $\phi_{i}$, then $\cos \left(2 \pi \nu_{i} t / T_{i}\right)$ in (5-10) can be replaced by $a_{i} \cos \left(2 \pi \nu_{i} t / T_{i}+\phi_{i}\right)$, and also the corresponding spectrum can be calculated.

\section{5-3. Bunched, Longitudinal}

In this section, we assume a simple case that the particles are randomly distributed in synchrotron oscillation frequency $\omega_{S_{i}}$, but there is no revolution frequency spread. Thus, the time domain function is,

$$
f(t)=\sum_{i=1}^{N} \sum_{n=-\infty}^{\infty} \delta\left(t-n T+\tau \sin \left(\omega_{S i} n T\right)\right)
$$

Similar to (3-9), the frequency spectrum is,

$$
F(\omega)=\omega_{0} \sum_{i=1}^{N} \sum_{k=-\infty}^{\infty} J_{k}(\omega \tau) \sum_{n=-\infty}^{\infty} \delta\left(\omega-n \omega_{0}-k \omega_{S i}\right)
$$

For the bandwidth of the $n$th harmonic and the $k$ th synchrotron sideband, we have the $\Lambda$ function as,

$$
\Lambda\left(\omega-n \omega_{0}-k \omega_{S i}\right)=\omega-n \omega_{0}-k \omega_{S 0}-k \Delta \omega_{s} / 2
$$

where $\omega_{S 0}$ and $\Delta \omega_{s} / 2$ are defined similarly to $\omega_{0}$ and $\Delta \omega / 2$ for the revolution frequency spread.

The equation (5-20) shows that for a given $n$, there are synchrotron side distributions located around $k \omega_{S 0}$, the bandwidth is determined by $k \Delta \omega_{S}$. As $k$ becomes larger, the bandwidth will be larger. It is noted that the bandwidth is not dependent on $n$. At 
$k=0$, the frequency band is simply a delta function.

To estimate the energy contained in each band, we first rewrite (5-18) as,

$$
f(t)=\sum_{i=1 n}^{N} \sum_{-=-\infty}^{\infty} \delta\left(t-n T+\tau \sin \left(\omega_{S i} n T+\phi_{S i}\right)\right)
$$

where $\phi_{S i}$ denotes the synchrotron oscillation phase distribution, which is assumed to be random. Using (2-7), the frequency spectrum is,

$$
F(\omega)=\omega_{0} \sum_{i=1 k=-\infty}^{N} \sum_{k}^{\infty} J_{k}(\omega) e^{j k \phi_{S i}} \sum_{n=-\infty}^{\infty} \delta\left(\omega-n \omega_{0}-k \omega_{S i}\right)
$$

Note that for the pulses located at $k=0$, there is no phase spread. Therefore, to calculate the energy contained in these pulses, the approach of (5-7) cannot be used. In fact the energy contained in the $n$th harmonic and $k=0$ pulses is

$$
<|F(\omega)|^{2}>_{n, 0}=<\omega_{0}^{2} \sum_{i=1}^{N} \sum_{p=1}^{N} J_{0}^{2}\left(n \omega_{0} \tau\right) \delta\left(\omega-n \omega_{i}\right) \delta\left(\omega-n \omega_{p}\right)>\approx \omega_{0}^{2} J_{0}^{2}\left(n \omega_{0} \tau\right) N^{2}
$$

which shows that the energy is proportional to the square of the number of the particles.

For the sidebands where $k \neq 0$, there is phase spread, and therefore the similar approach to (5-7) and (5-8) can be used. The result is that for $n$th harmonic and the $k$ th sideband, the energy contained in each band is,

$$
\left.<|F(\omega)|^{2}\right\rangle_{n, k} \approx \omega_{0}^{2} J_{k}^{2}\left(n \omega_{0} \tau\right) N
$$

which is determined by $J_{k}^{2}(\omega \tau), \omega_{0}^{2}$, and $N$, but independent of $n$. An example of such energy spectrum is shown in Fig.13a. A blow-up of the energy spectrum at the harmonics from 2 to 4 is shown in Fig.13b. Several phenomena discussed above can be observed.

Using the identity,

$$
\sum_{k=-\infty}^{\infty} J_{k}^{2}(x)=1
$$

the equation (5-24) becomes,

$$
\left.<|F(\omega)|^{2}\right\rangle_{n} \approx \omega_{0}^{2} \sum_{k=-\infty}^{\infty} J_{k}^{2}\left(n \omega_{0} \tau\right) N=\omega_{0}^{2} N
$$


In general, in addition to the revolution frequency spread and different initial phase for each particle, the synchrotron oscillation amplitude for each particle is also different. We may replace $\delta\left(t-n T+\tau \sin \omega_{S i} n T\right)$ in (5-18) by $\delta\left(t-n T_{i}+\tau_{i} \sin \left(\omega_{S i} n T_{i}+\phi_{S i}\right)\right)$, and using (2-7), (3-8) and (3-9) the corresponding spectrum can be calculated.

\section{5-4. Bunched, Transverse}

In this section, we consider the revolution frequency spread, the corresponding betatron tune spread, and the independent synchrotron oscillation frequency spread.

The time domain function is

$$
f(t)=\sum_{i=1}^{N} \cos \left(2 \pi \nu_{i} t / T_{i}\right) \sum_{n=-\infty}^{\infty} \delta\left(t-n T_{i}+\tau \sin \omega_{S i} n T_{i}\right)
$$

Using (5-11) and (5-19), the frequency spectrum is found,

$$
\begin{aligned}
F(\omega) & =\frac{\omega_{0}}{2}\left[\sum_{i=1 k=-\infty}^{N} \sum_{k}^{\infty} J_{k}\left(\omega r-\nu_{i} \omega_{i} \tau\right) \sum_{n=-\infty}^{\infty} \delta\left(\omega-n \omega_{i}-k \omega_{S i}-\nu_{i} \omega_{i}\right)\right. \\
& \left.+\sum_{i=1}^{N} \sum_{k=-\infty}^{\infty} J_{k}\left(\omega \tau+\nu_{i} \omega_{i} \tau\right) \sum_{n=-\infty}^{\infty} \delta\left(\omega-n \omega_{i}-k \omega_{S i}+\nu_{i} \omega_{i}\right)\right]
\end{aligned}
$$

The energy spectrum is shown in Fig.14. To determine the bandwidth and the energy contained in each sideband, the approach used previously can be applied. We will not repeat the calculation here. 


\section{References}

(1) D. Boussard, 'Schottky Noise and Beam Transfer Function Diagnostics', Proc. CERN Accelerator School, Advanced Accel. Physics, CERN 87-03, 1987.

[2] S. Chattopadhyay, 'Some Fundamental Aspects of Fluctuation and Coherence in Charged-Particle Beams in Storage Rings,' Proc. AIP Conference, No.127, pp.467$623,1985$.

[3] I. S. Gradshteyn and I. M. Ryshik, 'Table of Integrals, Series, and Products,' New York, Academic Press, 1965.

(4) R. Littauer, 'Beam Instrumentation', Proc. AIP Conference, No. 105, pp.869-952, 1982.

[5] A. Papoulis, 'The Fourier Integral and Its Applications,' New York, McGraw-Hill, 1962.

[6] R. H. Siemann, 'Bunched Beam Diagnostics' Proc. AIP Conference, No. 184, pp.430-471, 1989. 


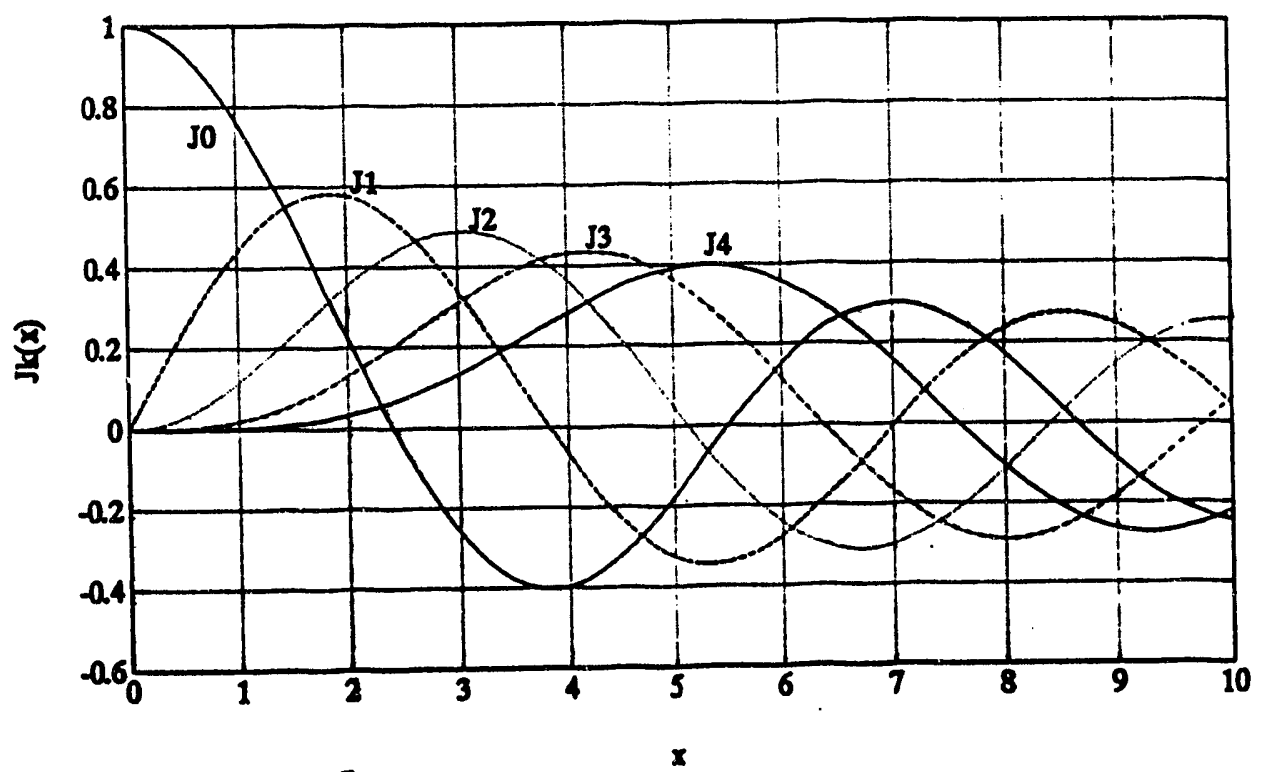

Fig.1. Bessel Functions. 


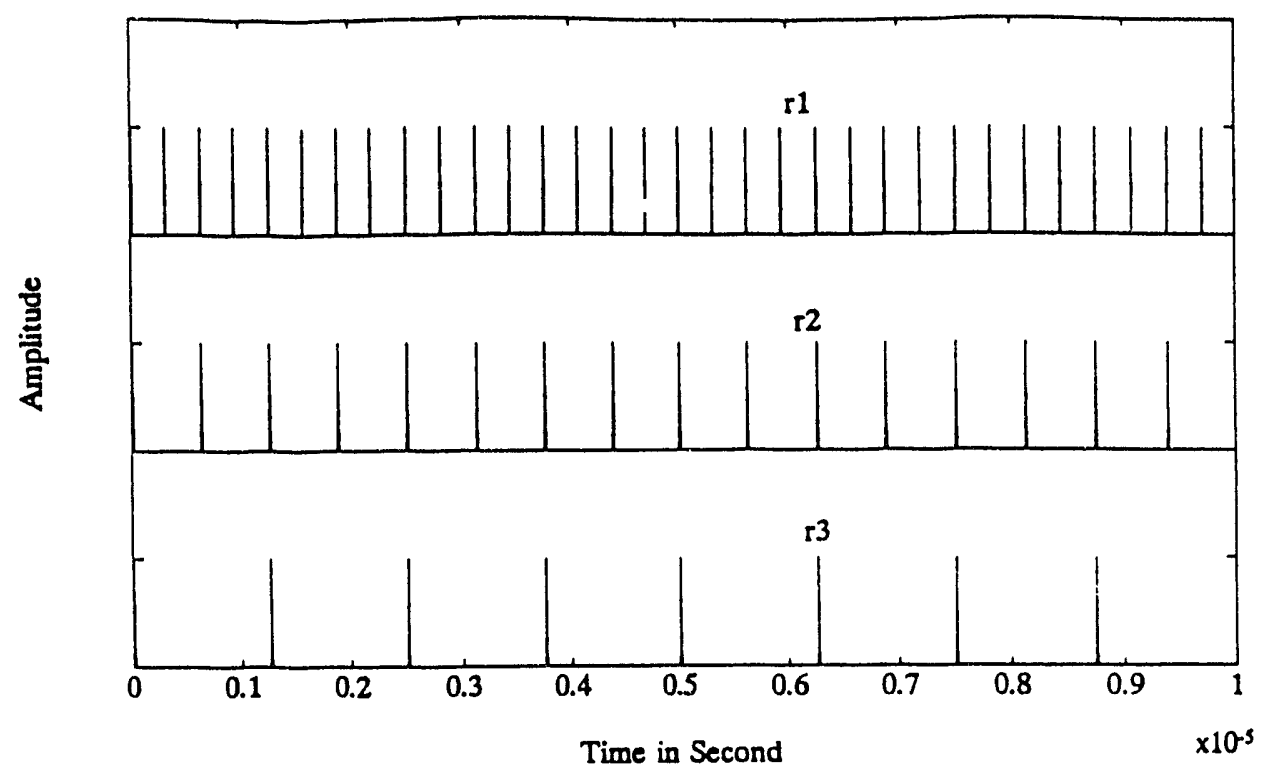

Fig.2a. Single Particle Signal, Longitudinal.

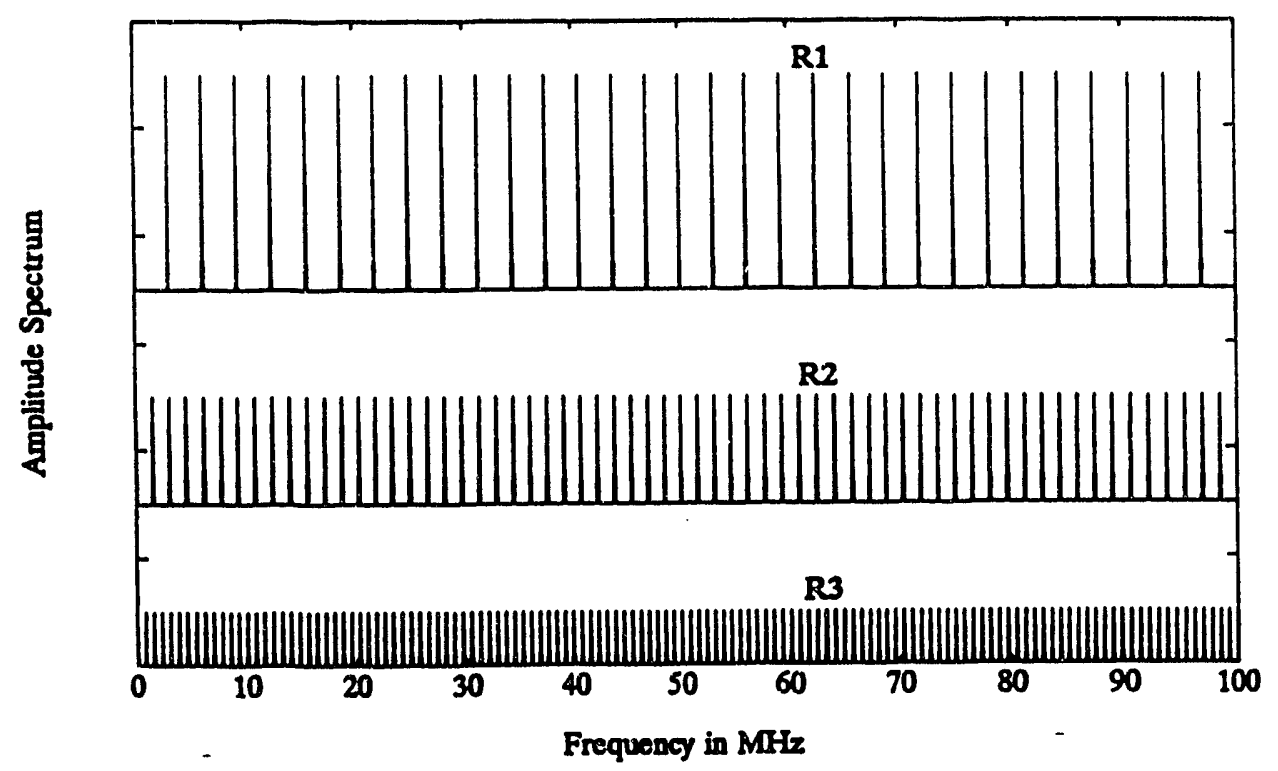

Fig.2b. Single Particle Amplitude Spectrum, Longitudinal. 


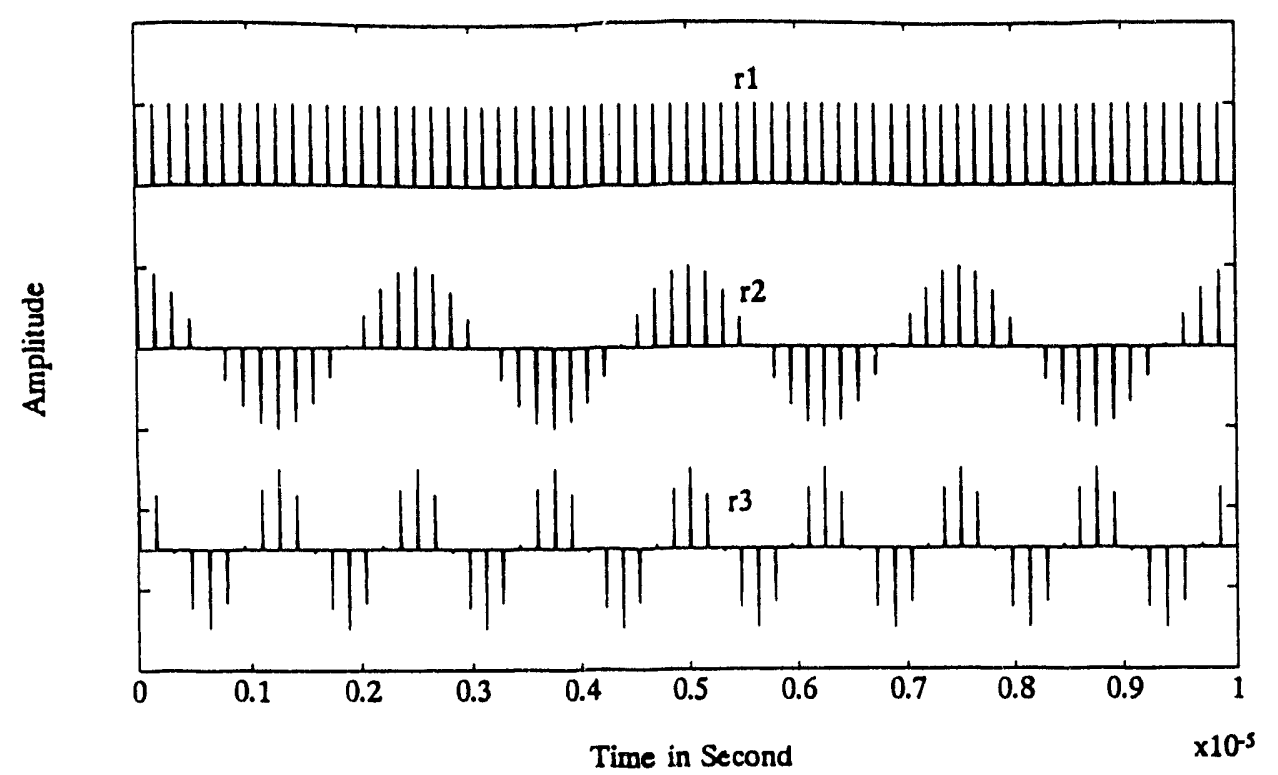

Fig.3a. Single Particle Signal, Transverse.

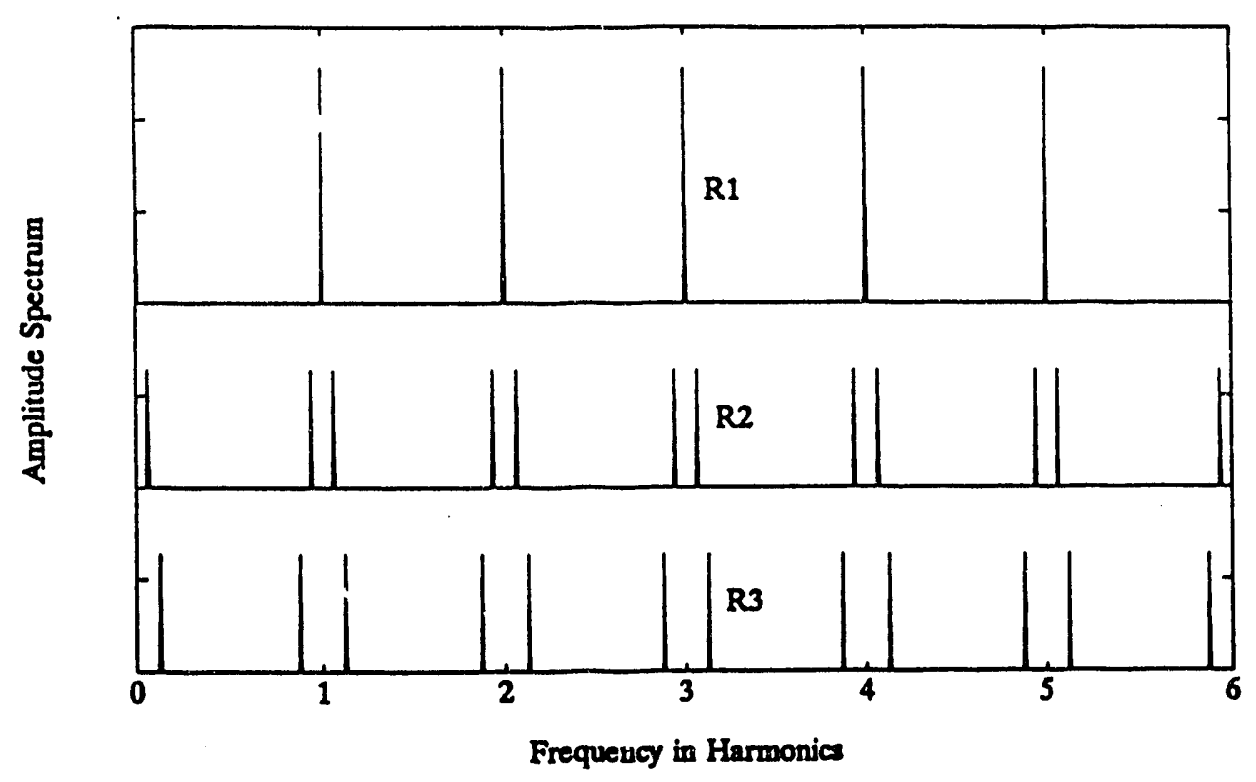

Fig.3b. Single Particle Amplitude Spectrum, Transverse. 


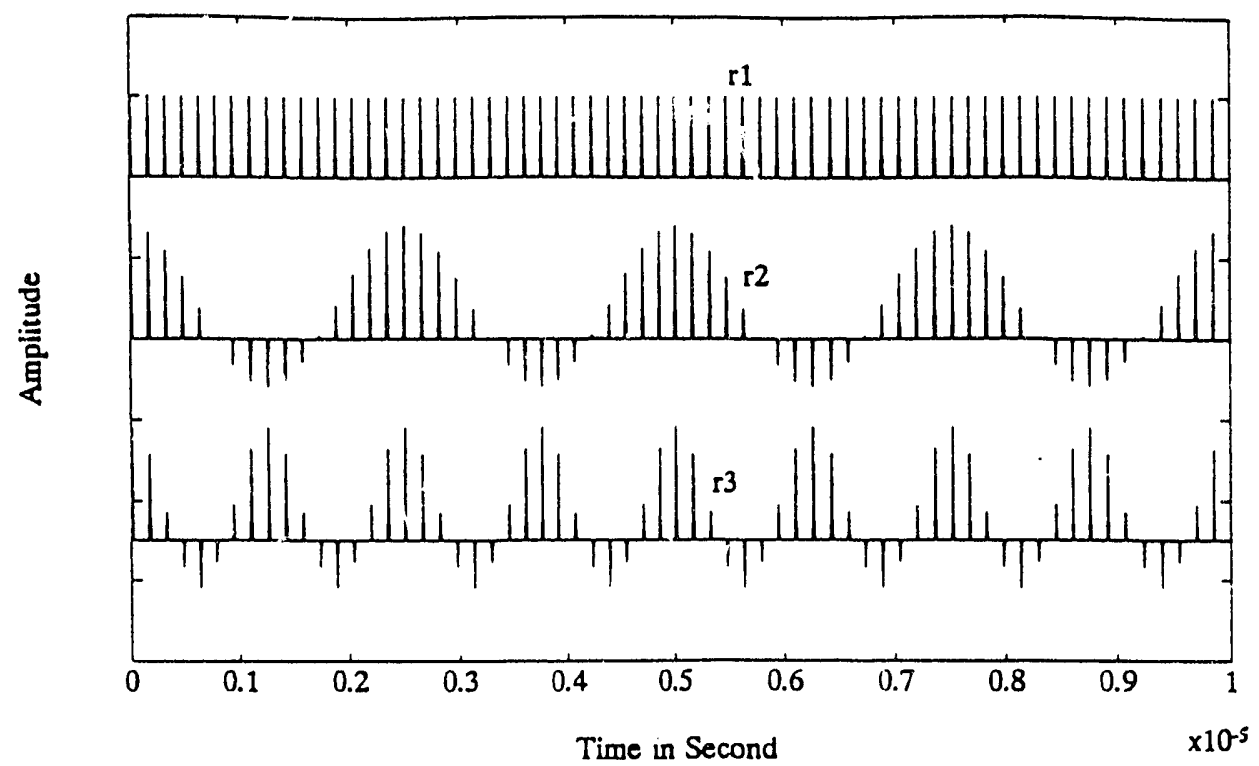

Fig.3c. Single Particle Signal, Transverse.

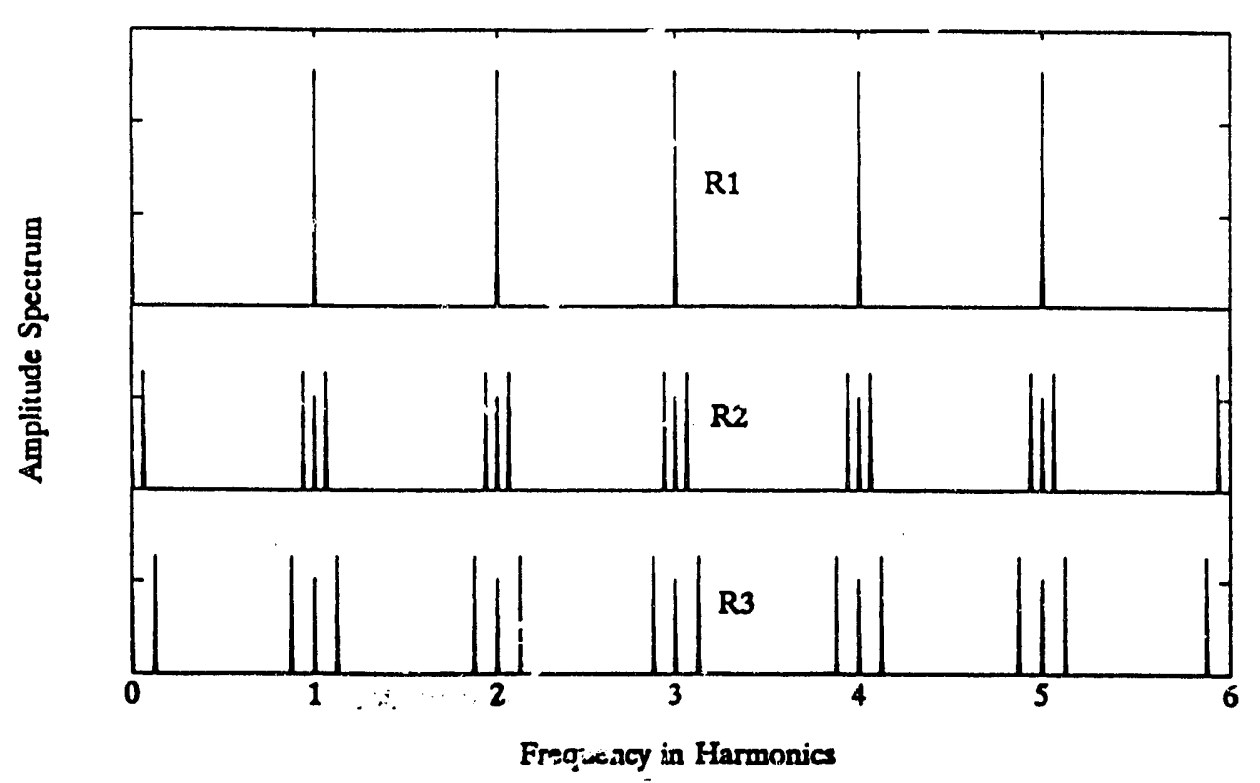

Fig.3d. Single Particle Amplitude Spectrum, Transverse. 


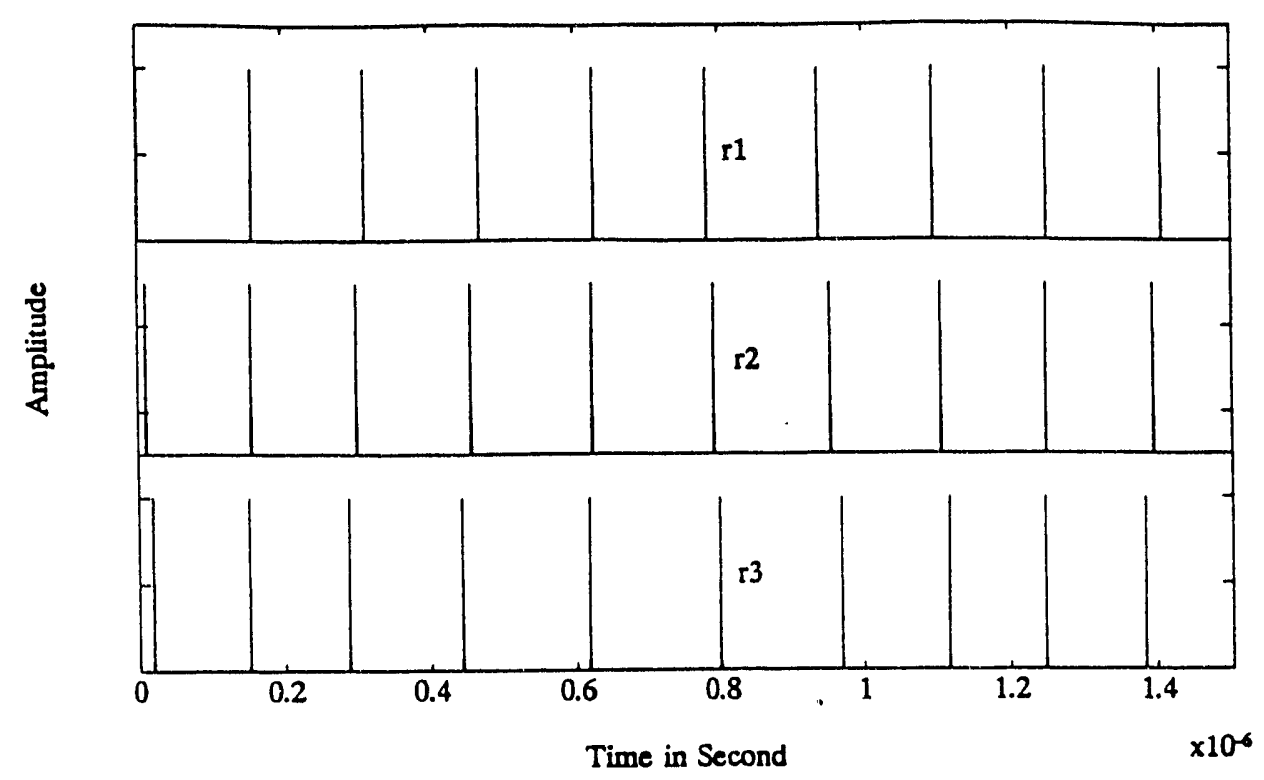

Fig.4a. Bunched Beam Signal, Longitudinal.

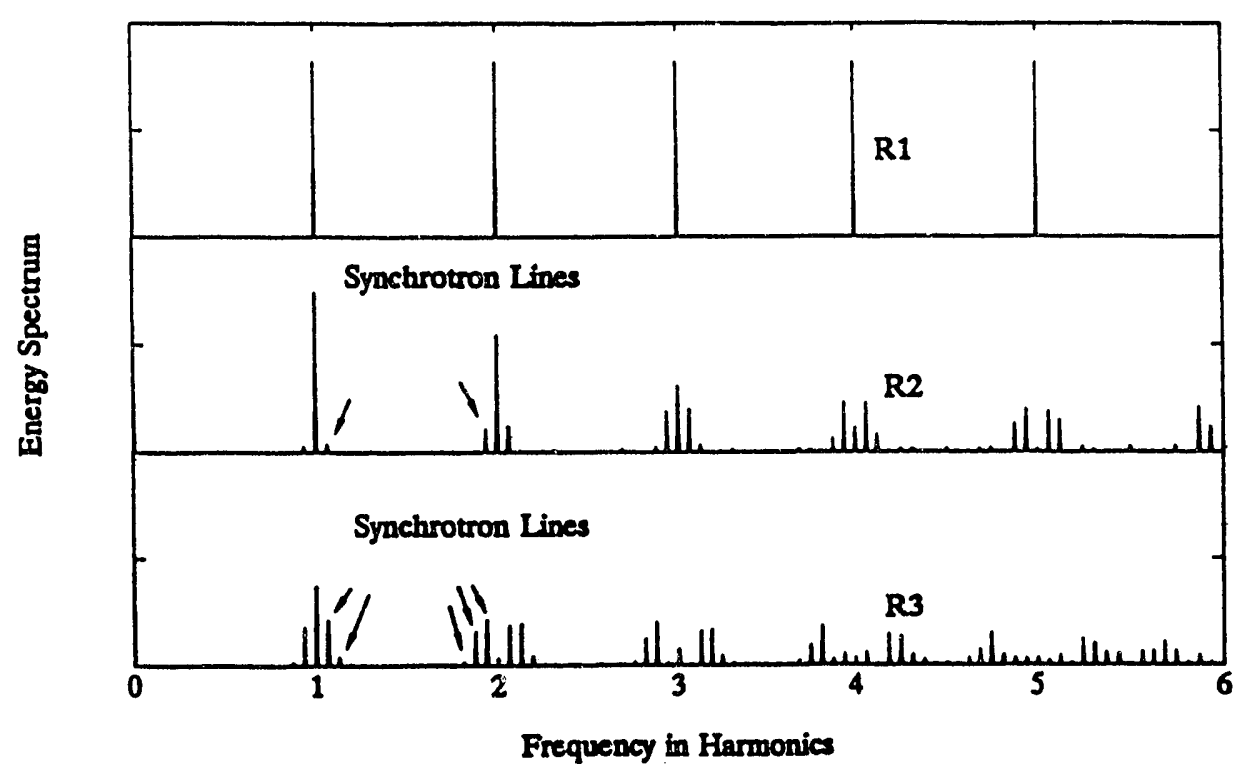

Fig.4b. Bunched Beam Energy Spectrum, Longitudinal. 


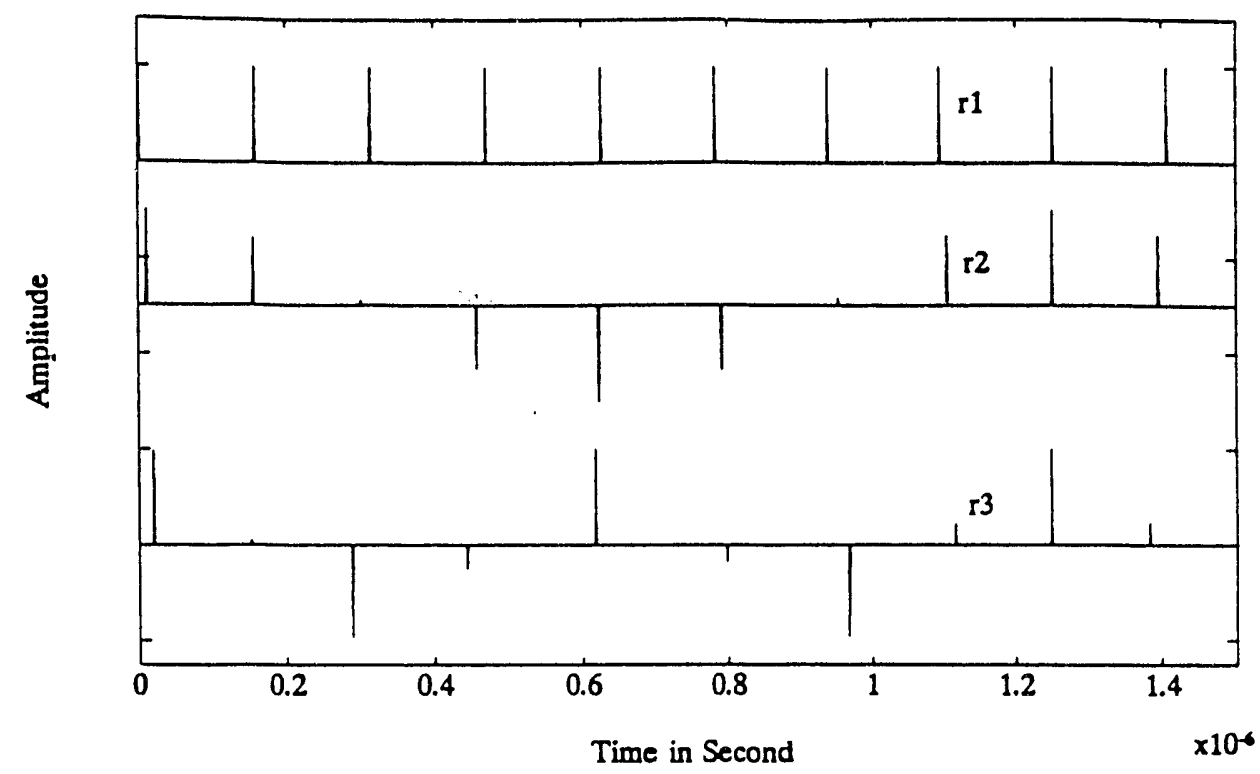

Fig.5a. Bunched Beam Signal, Transverse.

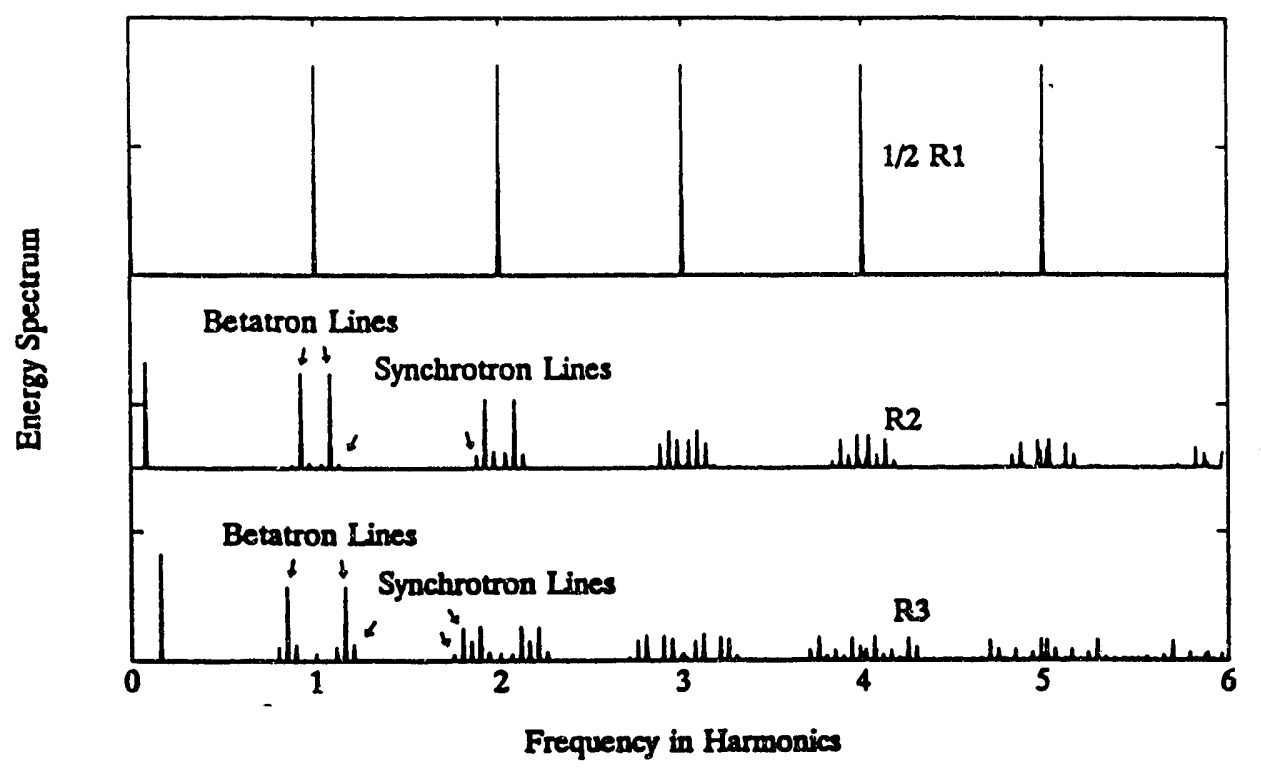

Fig.5b. Bunched Beam Energy Spectrum, Transverse. 


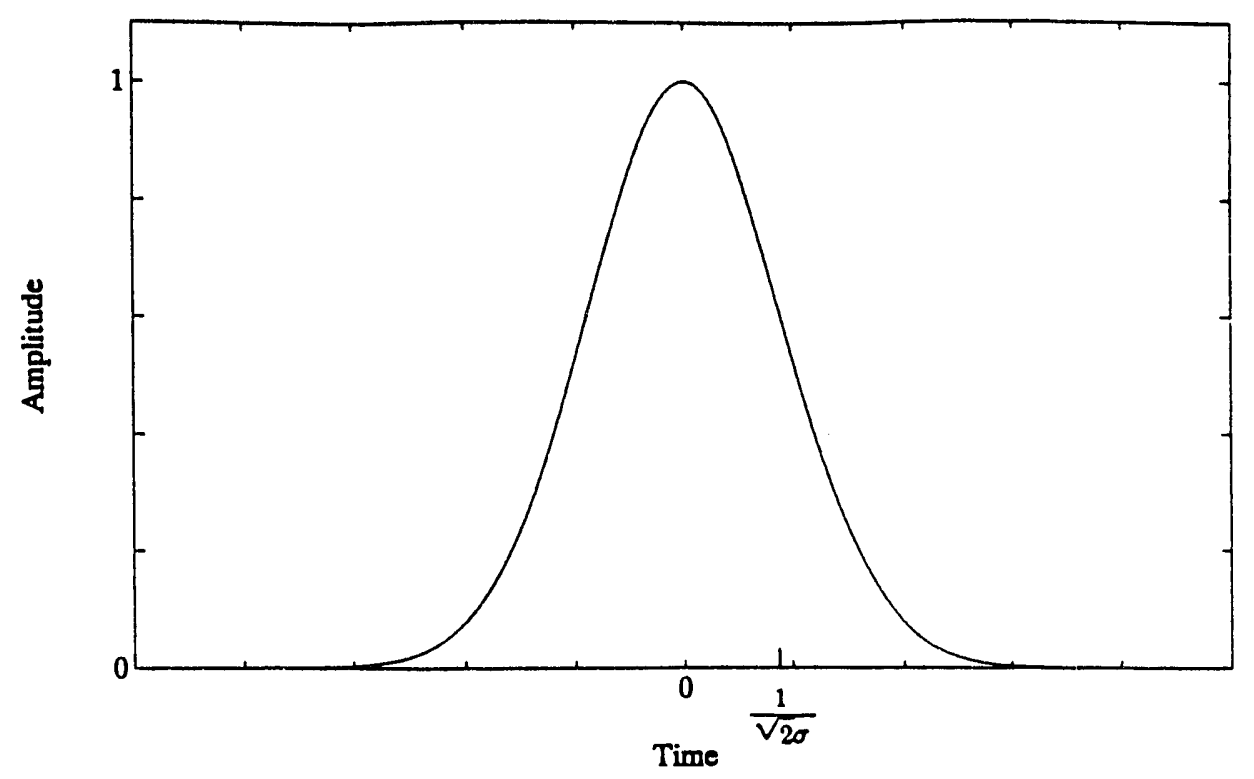

Fig.6a. Gaussian Distribution.

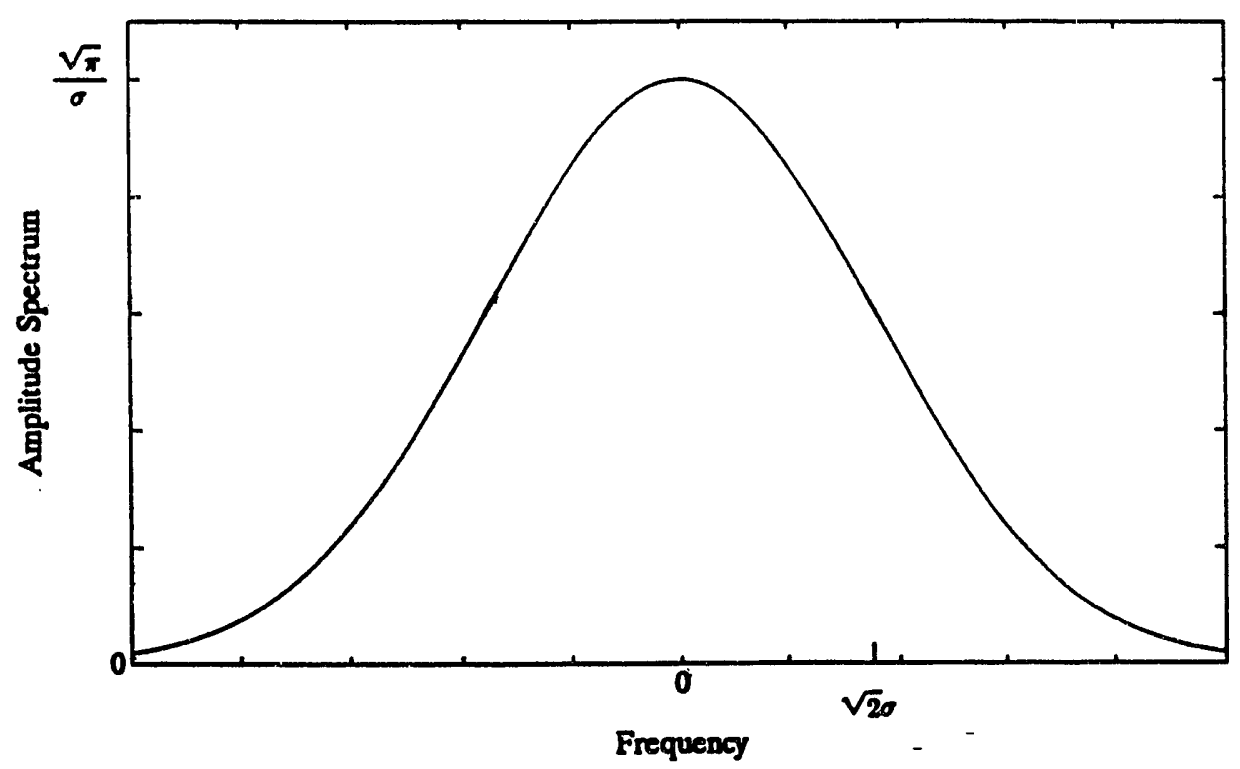

Fig.6b. Amplitude Spectrum of Gaussian Distribution. 


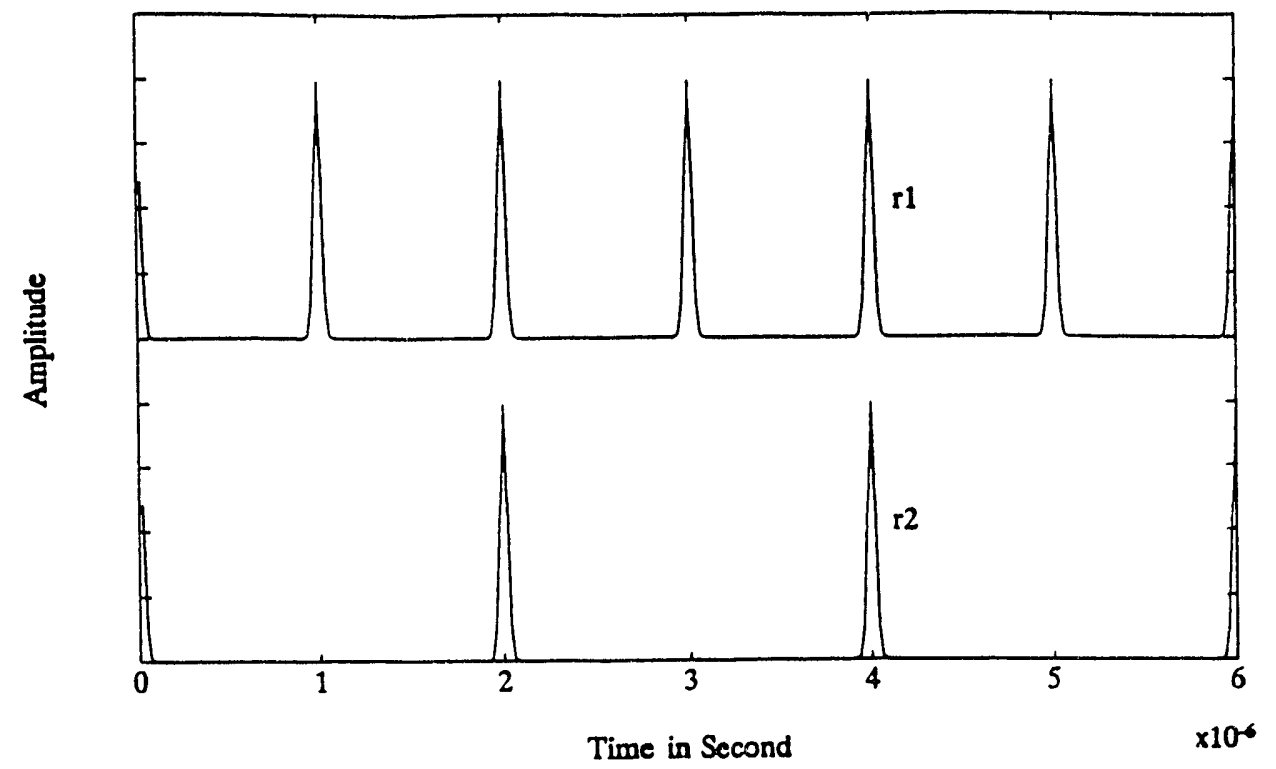

Fig.7a. Multiple Particle Signal, Longitudinal.

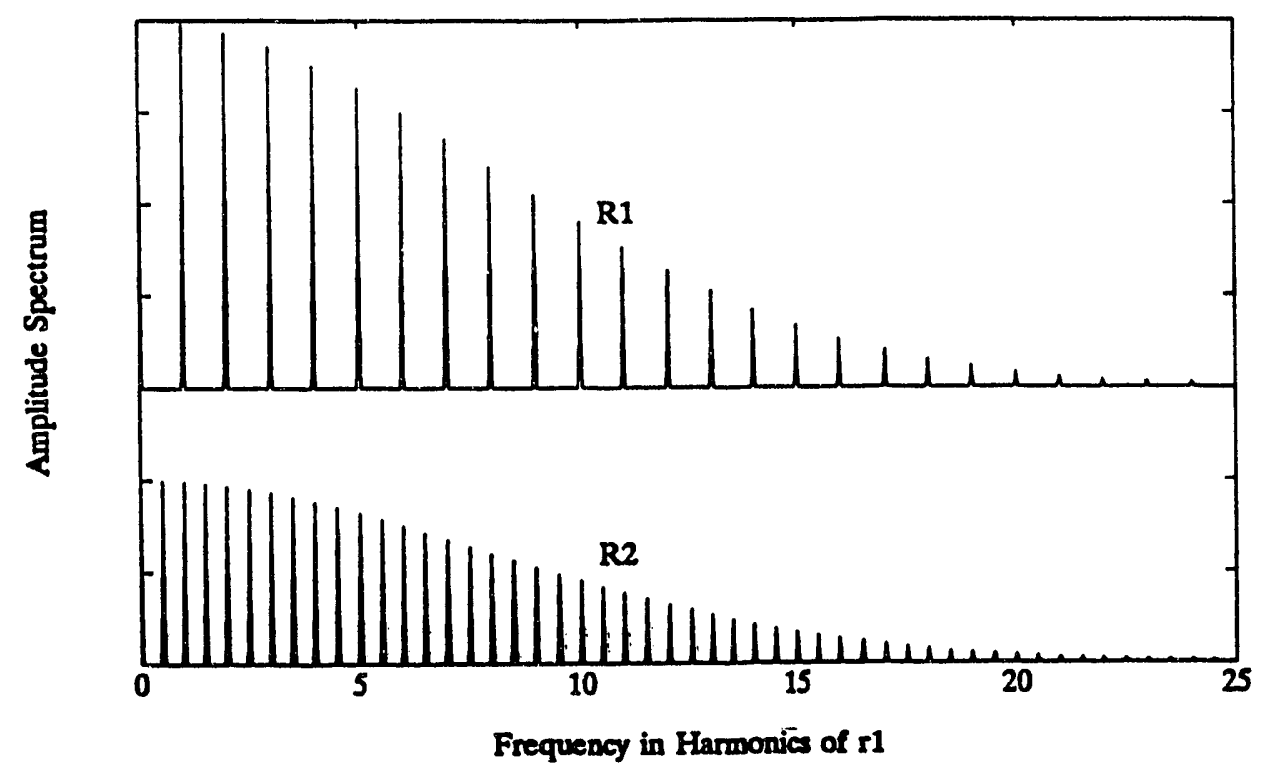

Fig.7b. Multiple Particle Amplitude Spectrum, Longitudinal. 


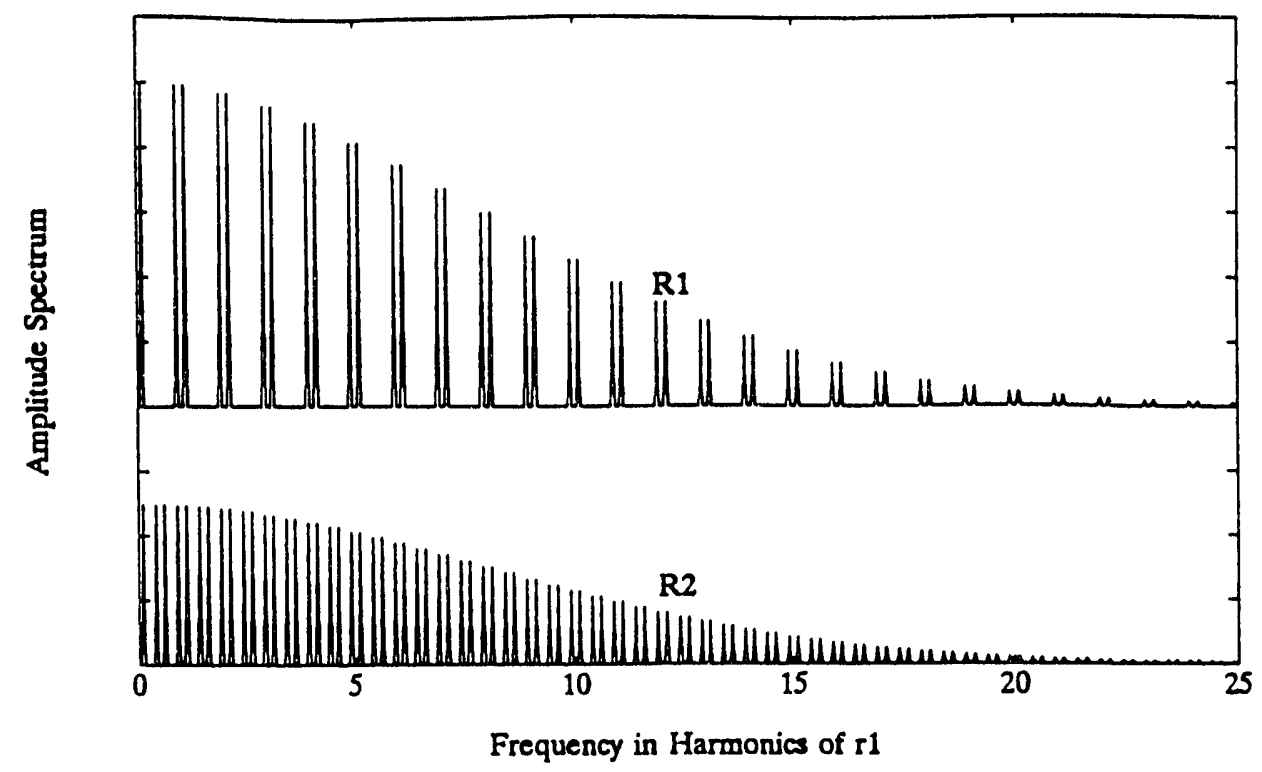

Fig.8. Multiple Particle Amplitude Spectrum, Transverse.

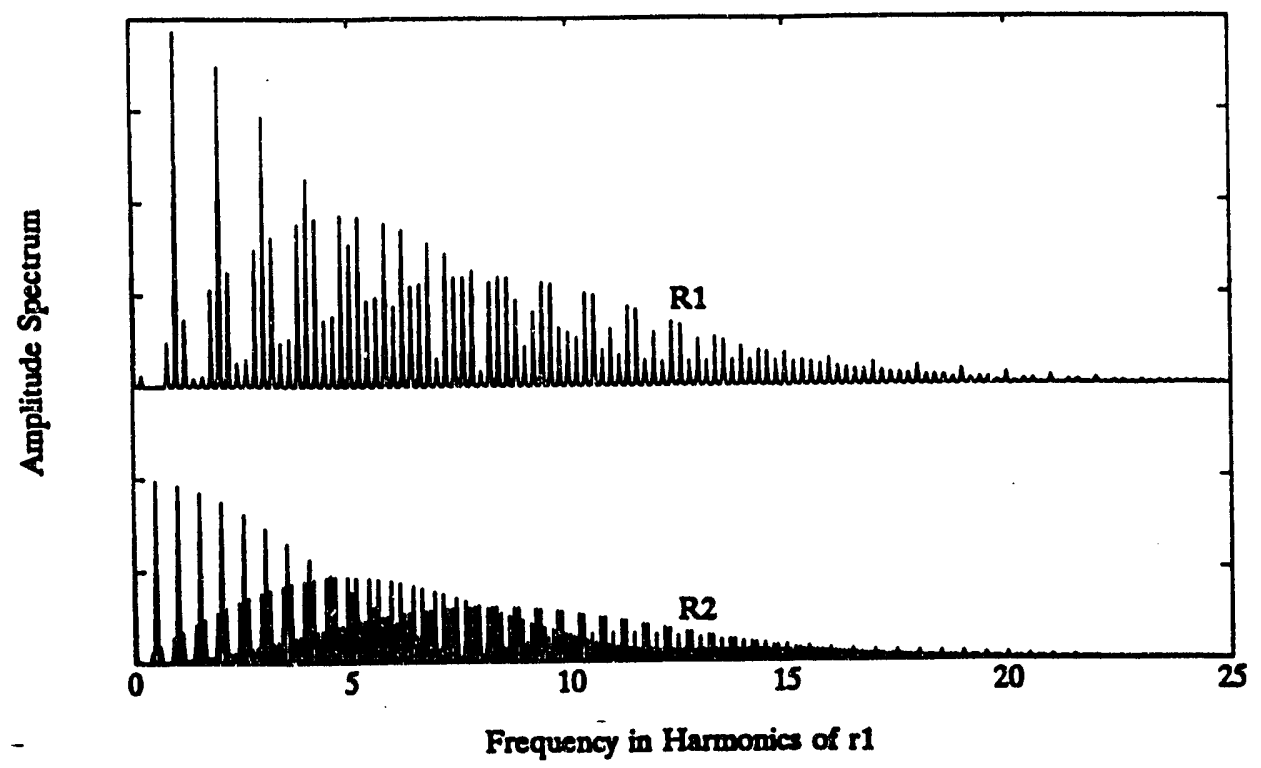

Fig.9. Multiple Particle Amplitude Spectrum, Bunched, Longitudinal. 


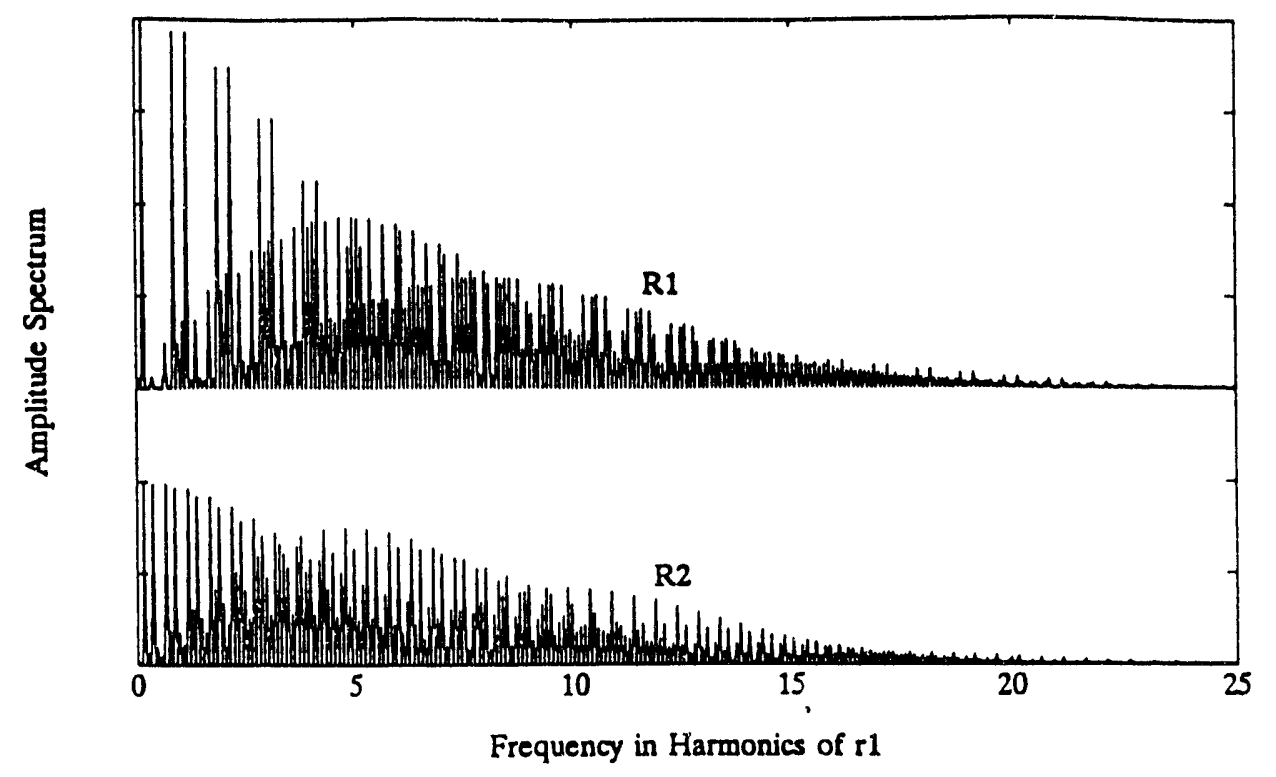

Fig.10. Multiple Particle Amplitude Spectrum, Bunched, Transverse.

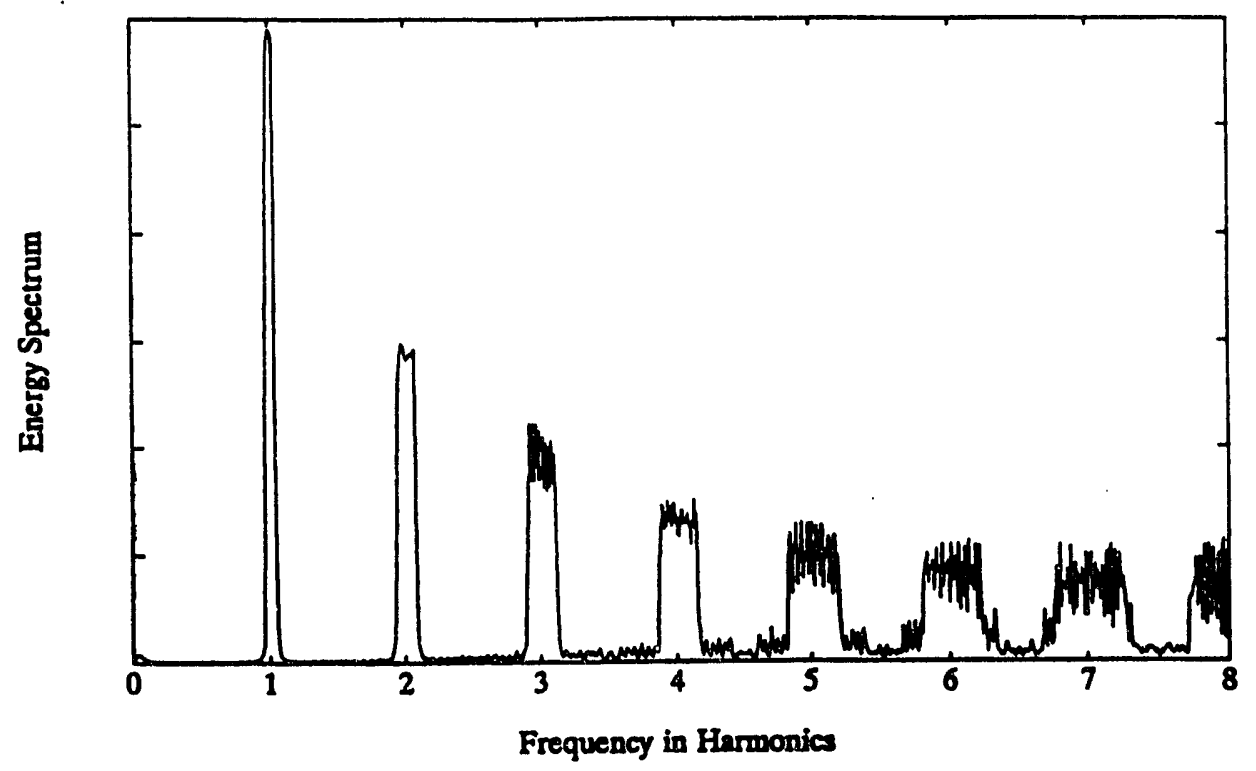

Fig.11. Schottky Noise Energy Spectrum, Longitudinal. 


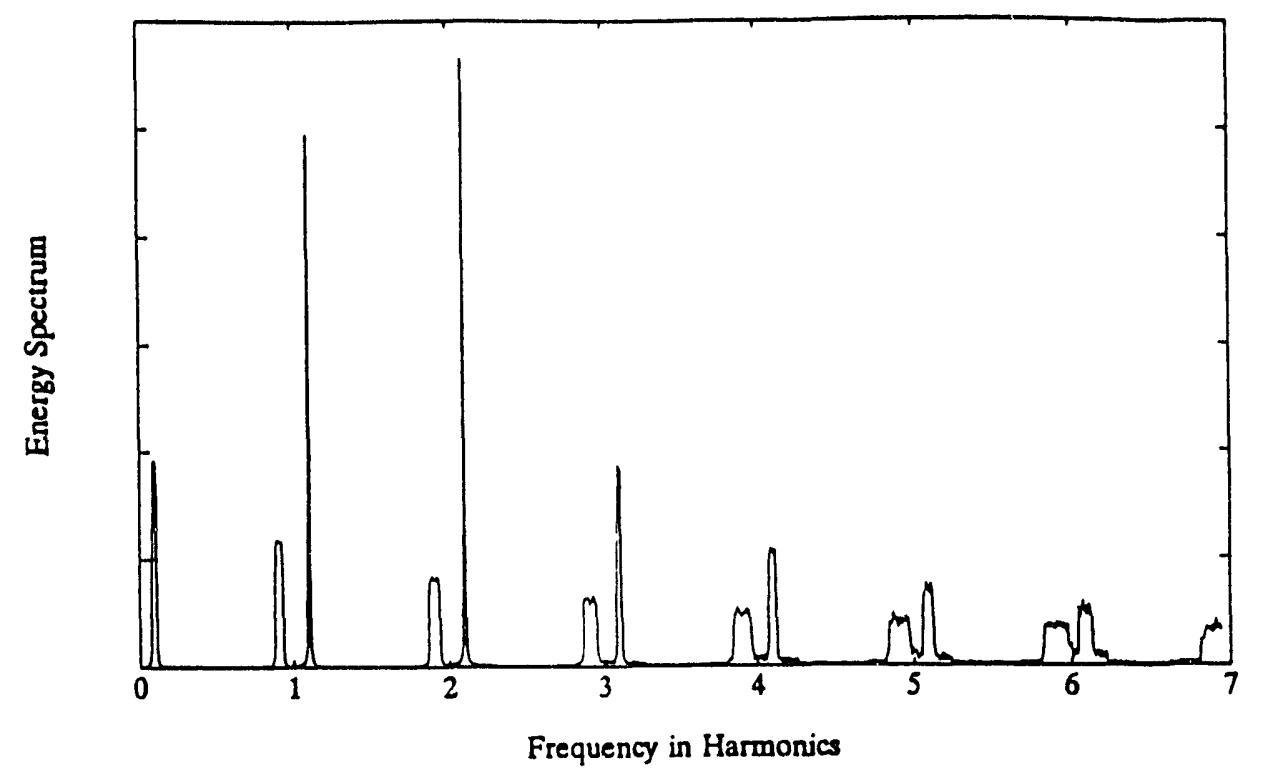

Fig.12. Schottky Noise Energy Spectrum, Transverse.

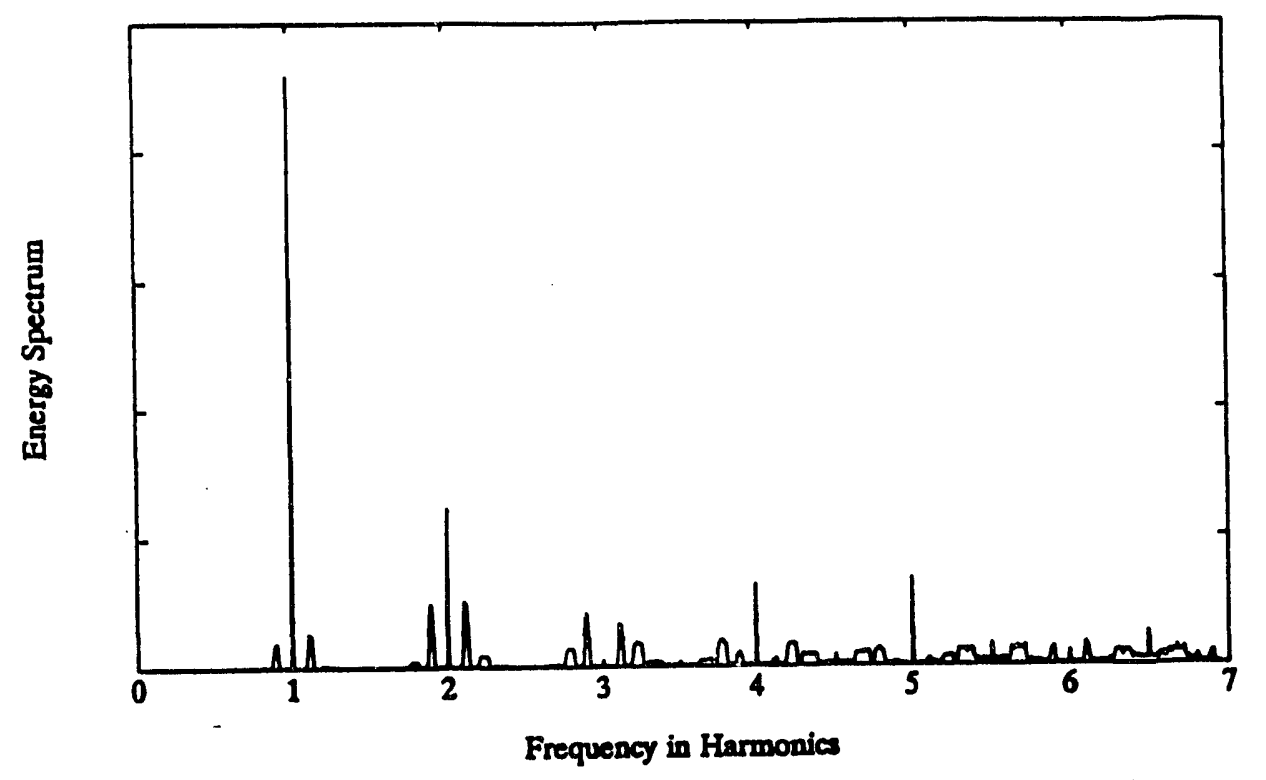

Fig.13a. Schottky Noise Energy Spectrum, Bunched, Longitudinal. 


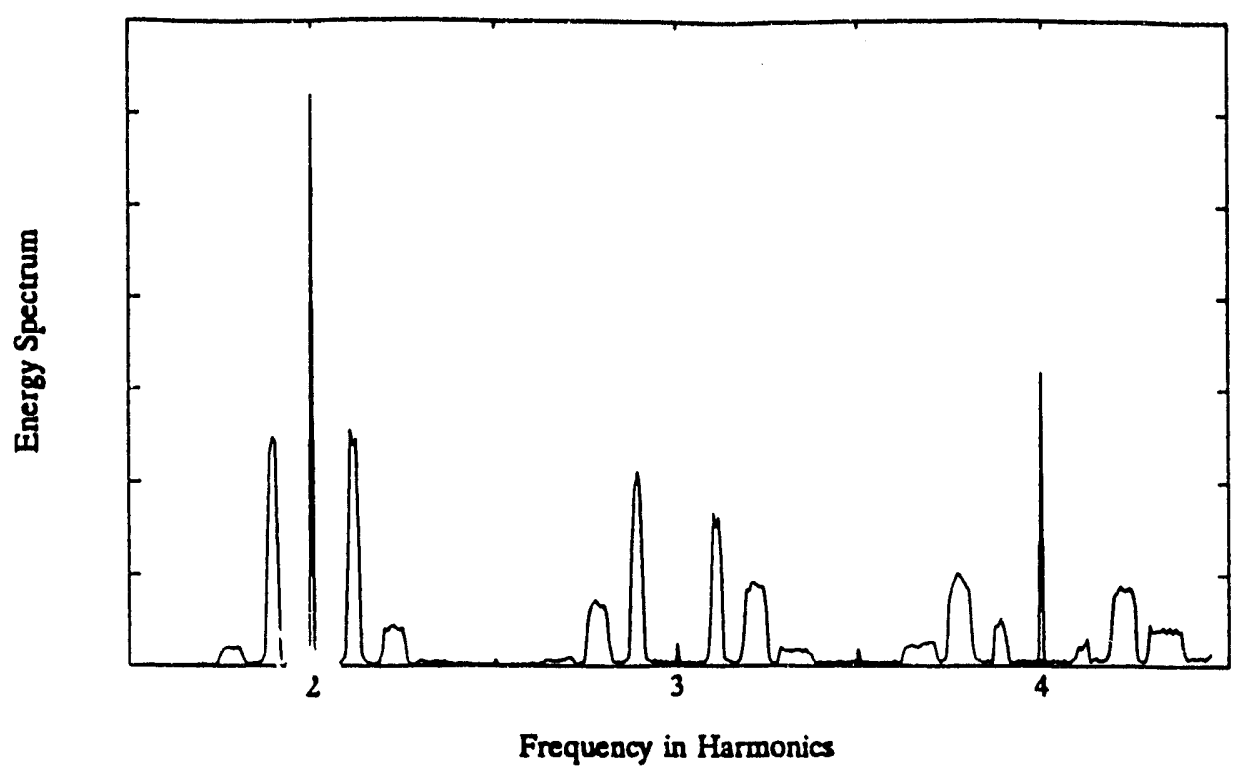

Fig.13b. Schottky Noise Energy Spectrum, Bunched, Longitudinal.

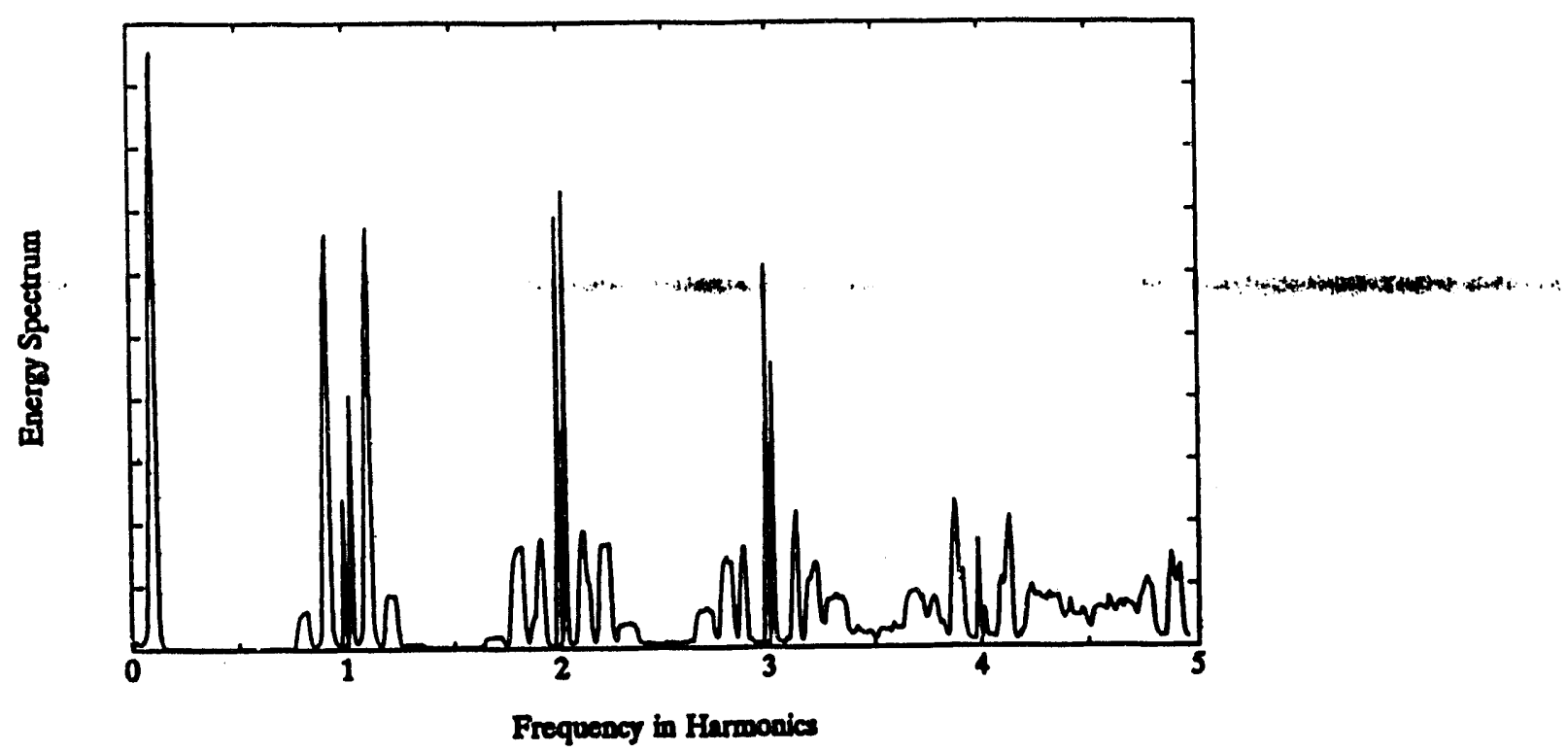

Fig.14. Schottky Noise Energy Spectrum, Bunched, Transverse. 

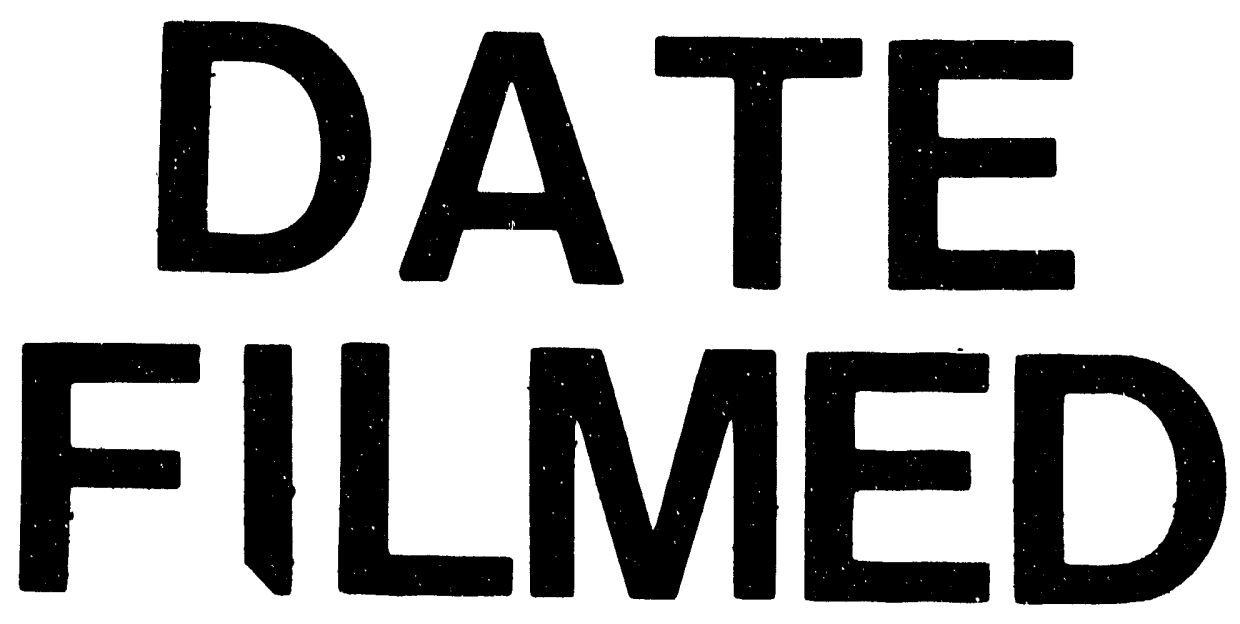

$7|14| 93$ 
